\title{
Neurochemical characterization of the tree shrew dorsal striatum
}

\section{Matthew W. Rice, Rosalinda C. Roberts, Miguel Melendez-Ferro* and Emma Perez-Costas*}

Department of Psychiatry and Behavioral Neurobiology, University of Alabama at Birmingham, Birmingham, AL, USA

Edited by:

Jose L. Lanciego, University of

Navarra, Spain

\section{Reviewed by:}

Jose L. Lanciego, University of

Navarra, Spain

Rosa M. Villalba, Emory University, USA

\section{*Correspondence:}

Emma Perez-Costas and Miguel Melendez-Ferro, Department of Psychiatry and Behavioral Neurobiology, University of Alabama at Birmingham, Sparks Center (SC865), 1720 7th Avenue South Birmingham, AL, 35294, USA. e-mail: epcostas@uab.edu; melendez@uab.edu

\begin{abstract}
The striatum is a major component of the basal ganglia and is associated with motor and cognitive functions. Striatal pathologies have been linked to several disorders, including Huntington's, Tourette's syndrome, obsessive-compulsive disorders, and schizophrenia. For the study of these striatal pathologies different animal models have been used, including rodents and non-human primates. Rodents lack on morphological complexity (for example, the lack of well defined caudate and putamen nuclei), which makes it difficult to translate data to the human paradigm. Primates, and especially higher primates, are the closest model to humans, but there are ever-increasing restrictions to the use of these animals for research. In our search for a non-primate animal model with a striatum that anatomically (and perhaps functionally) can resemble that of humans, we turned our attention to the tree shrew. Evolutionary genetic studies have provided strong data supporting that the tree shrews (Scadentia) are one of the closest groups to primates, although their brain anatomy has only been studied in detail for specific brain areas. Morphologically, the tree shrew striatum resembles the primate striatum with the presence of an internal capsule separating the caudate and putamen, but little is known about its neurochemical composition. Here we analyzed the expression of calcium-binding proteins, the presence and distribution of the striosome and matrix compartments (by the use of calbindin, tyrosine hydroxylase, and acetylcholinesterase immunohistochemistry), and the GABAergic system by immunohistochemistry against glutamic acid decarboxylase and Golgi impregnation. In summary, our results show that when compared to primates, the tree shrew dorsal striatum presents striking similarities in the distribution of most of the markers studied, while presenting some marked divergences when compared to the rodent striatum.
\end{abstract}

Keywords: caudate, putamen, GABAergic, dopaminergic, calbindin, calretinin, parvalbumin, striosomes

\section{INTRODUCTION}

The mammalian striatum is a major component of the basal ganglia and contains its major receptive nuclei, the caudate, and the putamen. These nuclei are the target of several areas of the mammalian brain including the cortex, mesencephalon, and thalamus (Kawaguchi et al., 1995; Joel and Weiner, 2000). In addition, the caudate and putamen send projections to other nuclei of the basal ganglia, such as the substantia nigra (SN) and globus pallidus (GP; Royce and Laine, 1984). There are also indirect projections to the subthalamic nucleus, thalamus, and cerebral cortex via the direct and indirect pathways (Smith et al., 1998).

The striatum receives inputs from the dopaminergic, glutamatergic, and serotonergic systems (Kubota et al., 1986; Lavoie and Parent, 1990; Fujiyama et al., 2006). In addition, some afferent projections of the striatum have been shown to synapse onto particular cytoarchitectonic components of the caudate and/or putamen (see as a review Nieuwenhuys et al., 2007). Even when there is no complete segregation of the target areas, the caudate and putamen present two distinguishable types of compartments with their own specific properties. These compartments are known as the striosomes (patch) and matrix, which receive preferentially different inputs and contain cells that express different markers
(Graybiel and Ragsdale, 1983; Donoghue and Herkenham, 1986; Bolam et al., 1988; Ragsdale and Graybiel, 1988; Gerfen, 1989; Hirsch et al., 1989; Graybiel, 1990; Kubota and Kawaguchi, 1993; Holt et al., 1997). Ventral to the caudate and putamen is an area of the striatum known as the "ventral striatum." Different nuclei have been suggested to be part of the ventral striatum (Heimer and Wilson, 1975; Heimer et al., 1982; Haber and McFarland, 1999; Fudge and Haber, 2002), of which the most prominent is the accumbens nucleus.

Medium spiny projection neurons are the most numerous type of cells in the dorsal striatum, with at least $75 \%$ of neurons belonging to this type in primates (Graveland and DiFiglia, 1985; see as reviews Nieuwenhuys et al., 2007; Tepper et al., 2010), and up to $95 \%$ in rodents and in the cat (Kemp and Powell, 1971; Graveland and DiFiglia, 1985; see as a review Voogd et al., 1998). These neurons are gamma-aminobutyric acid (GABA)ergic, and receive afferent connections from different neurotransmitter systems that modulate their activity by establishing synapses with them in a hierarchically and highly organized manner (Fonnum et al., 1981; Kubota et al., 1986; Dube et al., 1988; Lavoie and Parent, 1990; Parent and Hazrati, 1995). These GABAergic neurons are responsible for striatal efferent projections and can be 
subdivided into those containing enkephalin, that project to the external segment of the GP, and those containing substance P, that project to the internal segment of the GP and SN pars reticulata (Haber and Elde, 1982; Bolam and Izzo, 1988; Bolam and Smith, 1990; Reiner et al., 1999). The second class of neurons present in the dorsal striatum are interneurons (GABAergic or cholinergic), that are typically aspiny, and unlike the medium spiny neurons, do not send projections outside the striatum (Phelps et al., 1985; Cowan et al., 1990; Kawaguchi, 1993; Kawaguchi et al., 1995; Wu and Parent, 2000; see as reviews, Nieuwenhuys et al., 2007; Tepper et al., 2010). GABAergic interneurons can contain additional neurotransmitters such as parvalbumin (type I GABAergic striatal interneurons), somatostatin and neuropeptide Y (type II GABAergic striatal interneurons), or calretinin (type III GABAergic striatal interneurons; DiFiglia and Aronin, 1982; Vincent et al., 1983; Cowan et al., 1990; Kita et al., 1990; Kawaguchi, 1993; Kubota et al., 1993; Kawaguchi et al., 1995; Rushlow et al., 1995; Parent et al., 1996; Kubota and Kawaguchi, 2000; Wu and Parent, 2000; see as reviews, Nieuwenhuys et al., 2007; Tepper et al., 2010). Finally, the striatum also contains a small amount of dopaminergic intrinsic neurons. Although the number of these neurons is almost vestigial in normal rodent striatum, it is more prevalent in the primate striatum (Dubach et al., 1987; Tashiro et al., 1989; Mura et al., 1995; Ikemoto et al., 1996; Betarbet et al., 1997; Baker et al., 2003; Cossette et al., 2004, 2005; Huot and Parent, 2007).

The caudate and putamen nuclei are involved in motor and cognitive functions (DeLong and Georgopoulos, 1981; DeLong et al., 1984; Middleton and Strick, 1994). Disruptions in the afferent and efferent connections and alterations within the nuclei themselves have been linked to motor disorders such as Parkinson's and Huntington's disease (DeLong and Georgopoulos, 1981; DeLong et al., 1984; Marsden, 1986), and psychiatric disorders such as schizophrenia (Buchsbaum et al., 1992; Hietala et al., 1995; Roberts et al., 1996, 2005; Howes et al., 2009; see also as reviews Perez-Costas et al., 2010; Simpson et al., 2010), bipolar disorder (Amsterdam and Newberg, 2007; Cousins et al., 2009), and major depression (Matsuo et al., 2008; Butters et al., 2009; Khundakar et al., 2011). Although some aspects of these diseases can be studied in postmortem human tissue, the use of animal models is also necessary. Animal models allow the study of pathological states in a controlled environment, and the test of experimental treatments. The majority of animal research involves the use of rodents, and the results obtained from these studies have provided a large body of data, although their significance is limited due to the anatomical differences between the rodent and the human striatum. In the search for a striatum that anatomically (and possibly functionally) presents more similarities to that of the human, many researchers have used non-human primates for their studies. The use of non-human primates provides a more accurate model of the human basal ganglia, but these studies tend to be costly and create ethical controversy. This has moved some countries to partially or totally ban research in some groups of primates (Cohen, 2007a,b; Vogel, 2010). All of these factors raise the need for alternative models that can represent a closer frame for comparison to the human striatum than rodents, and at the same time do not incur the cost and controversy associated with primate research. One viable option that may fit this compromise is the common tree shrew (Tupaia glis belangeri). Tupaiids are diurnal omnivorous mammals that in their natural environment occupy arboreal, semi-arboreal, and forest floor habitats. These animals are able to use their forepaws to climb trees and handle food (see Luckett, 1980). Evolutionary genetic studies using sequencing of large DNA sets (mitochondrial and nuclear) have provided strong data supporting that Scadentia (tree shrews) and Dermoptera (flying lemurs) are the closest groups to primates, with a common ancestor far removed from other groups such as Lagomorpha (rabbits and pikas) or Chiroptera (bats; Adkins and Honeycutt, 1991; Liu et al., 2001; Murphy et al., 2001; Janecka et al., 2007). Behavioral studies have demonstrated that tree shrews have the capability to develop strategic planning (Bartolomucci et al., 2001), and when subjected to psychosocial stress they develop all the hallmarks of depression (behavioral, molecular, and anatomical), that in a similar manner to humans, are reversed with the use of antidepressants (Fuchs et al., 1995; Gould et al., 1997; Isovich et al., 2000; Czeh et al., 2001; Fuchs and Flugge, 2002; Fuchs, 2005). In addition, a recent study has shown that as it occurs in elderly humans, aged tree shrews present with accumulation of amyloid beta proteins, a pathology that has not been observed in aged wild-type mice (Dickson et al., 1992; Yamashita et al., 2010).

The tree shrew brain anatomy has only been studied in detail for pathways related to the visual system (see for example Fitzpatrick, 1996; Lyon et al., 2003; Elston et al., 2005; McCoy et al., 2008; Poveda and Kretz, 2009; Chomsung et al., 2010; Day-Brown et al., 2010). Some scarce studies have focused their interest in the general subdivisions of the neocortex (Remple et al., 2007; Wong and Kaas, 2009) as well as in punctual aspects of other brain areas including the striatum (see, e.g., Divac and Passingham, 1980; Oliver, 1982; Lin et al., 1984; Mijnster et al., 1999; Isovich et al., 2000; Keuker et al., 2003; Day-Brown et al., 2010). However, the tree shrew dorsal striatum has not been previously studied in detail, which is necessary to determine the suitability of this animal as a model for studies concerning striatal pathologies.

In this work we used histological stains as well as immunohistochemistry for markers known to be present in the dorsal striatum of humans and other mammalian species. The study of these markers allowed us the examination of chemoarchitectonic features, such as the presence of matrix and striosome compartments, and the distribution of certain neuronal cell types (for example, the presence of cells expressing different calcium-binding proteins). We also assessed the distribution of two of the main neurotransmitters in the striatum, GABA, and dopamine, by studying the expression of their synthesizing enzymes, glutamic acid decarboxylase (GAD), and tyrosine hydroxylase (TH). Finally, we used Golgi impregnation to provide a detailed description of the features of the main cell type of the striatum, the medium spiny neurons.

\section{MATERIALS AND METHODS ANIMALS AND TISSUE PREPARATION}

A total of 10 adult (1-5 years old) common tree shrews ( $T$. glis belangeri) were used in this study. Animals were kept and euthanized following protocols approved by the University of Alabama at Birmingham Institutional Animal Care and Use Committee 
(IACUC). For all the experiments, tree shrews were deeply anesthetized with a ketamine/xylazine mixture $(200,0.5 \mathrm{mg} / \mathrm{kg})$. For western-blot, animals were immediately decapitated and their brains were extracted and preserved at $-80^{\circ} \mathrm{C}$. Brain homogenates were obtained by sonication in a cold lysis buffer mixture (1:5 w:v) containing $50 \mathrm{mM}$ Tris pH 8.0, $5 \mathrm{mM}$ EDTA, $150 \mathrm{mM} \mathrm{NaCl}, 1 \%$ SDS, and $10 \mu \mathrm{l} / \mathrm{ml}$ of a protease inhibitor cocktail (Sigma, St. Louis, MO, USA; P8340). Samples were then centrifuged at $15000 \mathrm{~g}$ for $15 \mathrm{~min}$ at $4^{\circ} \mathrm{C}$, the supernatant was collected, and total protein concentration was measured using a modified Lowry technique (Bio-Rad, Hercules, CA, USA; D $\mathrm{C}$ Protein Assay; 500-0113, 5000114). Aliquots of $60 \mu \mathrm{g}$ of total protein were stored at $-80^{\circ} \mathrm{C}$. For immunohistochemistry and Nissl stain, tree shrews were perfused with a $0.9 \%$ saline solution followed by a cold $4 \%$ paraformaldehyde solution in $0.1 \mathrm{M}$ phosphate buffer $\mathrm{pH} 7.4$ (PB). The tissue was then immersed in a 30\% sucrose solution in PB for cryoprotection. Finally, six free-floating coronal parallel series of sections ( $50 \mu \mathrm{m}$ thick) were obtained on a cryostat, collected in a cryoprotection solution (FD NeuroTechnologies, Ellicott City, MD, USA; PC101) and stored at $-20^{\circ} \mathrm{C}$ until use. For Golgi impregnation, tree shrews were perfused only with a $0.9 \%$ saline solution and their brains were removed and immediately immersed in the impregnation solution.

In addition, a stock of striatal protein extracts from adult male Sprague-Dawley rat routinely maintained in our laboratory was used for western-blot studies. Finally, $50 \mu \mathrm{m}$ thick sections of adult male rat striatum from a stock of tissue kept in the laboratory were used as positive controls to test acetylcholinesterase antibodies.

\section{NISSL STAIN AND GOLGI IMPREGNATION}

The first whole series of each animal was stained with a standard thionin (Nissl) stain protocol, and was used as a reference series for morphology and landmark determination. To analyze in further detail the morphology of medium spiny neurons of the tree shrew striatum, Golgi-Cox impregnation was performed as described in Melendez-Ferro et al. (2009). Briefly, after perfusion with a 0.9\% saline solution, hemisected brains were immersed for 2 weeks in an impregnation solution that contained mercury chloride, potassium dichromate, and potassium chromate. After impregnation, the brains were immersed in a cryoprotectant solution at $4^{\circ} \mathrm{C}$ for a minimum of 1 week, frozen in dry ice, and after that, $150 \mu \mathrm{m}$ thick sections were obtained on a sliding microtome. Sections were collected on gelatin-subbed slides and allowed to dry for 5-6 days at $35^{\circ} \mathrm{C}$ on a warm plate. Development of the sections was achieved by incubation for $10 \mathrm{~min}$ at room temperature (RT) in a solution that contained ammonium hydroxide. Finally, sections were rinsed in distilled water, dehydrated in ethanol, cleared in xylene, and coverslipped using Eukitt (Electron Microscopy Sciences, PA, USA; 15322).

\section{ANTIBODIES USED IN THIS STUDY}

Antibodies against calbindin, parvalbumin, and calretinin were used to study the distribution of calcium-binding proteins within the caudate and putamen. The dopaminergic innervation was analyzed by the detection of tyrosine hydroxylase $(\mathrm{TH})$, the ratelimiting enzyme for the production of dopamine. The striatal GABAergic system was studied using an antibody against the two isoforms of glutamic acid decarboxylase (GAD65/67), the rate-limiting enzyme for the production of GABA. In addition, anti-acetylcholinesterase antibodies were used to further analyze the striosome/matrix organization in the tree shrew striatum. For each antibody a minimum of three whole series of different animals were studied.

\section{WESTERN-BLOT}

The specificity of the antibodies used in this study has been demonstrated by the manufacturers for different vertebrate species, although none of these antibodies have been characterized in the tree shrew. To ensure that these antibodies also recognized a protein of the appropriate molecular weight in the tree shrew brain, a western-blot analysis was performed using polyacrylamide gel electrophoresis in denaturing conditions. For this, samples were diluted $1: 1$ in loading buffer $(0.5 \mathrm{M}$ Tris- $\mathrm{HCl} \mathrm{pH} 6.8$, glycerol, $10 \%$ SDS, $0.5 \%$ bromophenol blue, and $\beta$-mercaptoethanol) and were heated to $95^{\circ} \mathrm{C}$ in a dry bath for $5 \mathrm{~min}$ before loading in a 4-20\% polyacrylamide gradient gel (Lonza, Basel, Switzerland; 58505). In each lane $60 \mu \mathrm{g}$ of tree shrew whole brain protein extract or $30 \mu \mathrm{g}$ of rat striatum protein extract were loaded in parallel. A molecular weight marker (Lonza; 50550) was also loaded for molecular weight reference. Proteins were resolved using a constant current of $125 \mathrm{~V}$, and then transferred to polyvinylidene fluoride (PVDF) membranes (Bio-Rad; 162-0714) using a constant current of $30 \mathrm{~V}$ for $19 \mathrm{~h}$ at $4^{\circ} \mathrm{C}$.

The following primary antibodies were used for western-blot: mouse monoclonal anti-tyrosine hydroxylase (TH; Millipore, Billerica, MA, USA; MAB5280, diluted 1:4000), mouse monoclonal anti-Calbindin (Sigma; C9848, diluted 1:8000), mouse monoclonal anti-Parvalbumin (Millipore; MAB1572, diluted 1:4000), rabbit polyclonal anti-GAD 65/67 (Millipore; AB1511, diluted 1:32000), and rabbit polyclonal anti-Calretinin (Swant, Bellinzona, Switzerland; $7699 / 3 \mathrm{H}$, diluted 1:32000). For all the antibodies tested, western-blot membranes were rinsed in $0.05 \mathrm{M}$ Tris Buffered Saline (TBS) and blocked with a solution containing $5 \%$ non-fat dry milk (Bio-Rad; 170-6404) in TBS containing $0.1 \%$ Tween 20 (TBS-T) for $1 \mathrm{~h}$ at RT. The membranes were then incubated with the appropriate primary antibody at the concentrations listed above in a solution of TBS-T with $1 \%$ non-fat dry milk for $19 \mathrm{~h}$ at $4^{\circ} \mathrm{C}$. After that, the membranes were rinsed in TBS-T containing 1\% non-fat dry milk prior to incubation with the appropriate secondary antibody, either alkaline phosphataseconjugated goat anti-mouse (Millipore; AP124A, diluted 1:15000) or alkaline phosphatase-conjugated goat anti-rabbit (Vector Laboratories, Burlingame, CA, USA; AP-1000, diluted 1:1000) in 1\% non-fat dry milk in TBS-T for $1 \mathrm{~h}$ at RT. The membranes were then rinsed and developed using an Immun-Star chemiluminescence kit (Bio-Rad alkaline phosphate substrate; 170-5018) and Kodak Biomax XAR films (Kodak, Rochester, NY, USA; 166-0760).

\section{IMMUNOHISTOCHEMISTRY}

In order to characterize the neurochemical composition of the tree shrew striatum, the same antibodies that were used for western-blot were also used for immunohistochemistry. The antibody concentrations used for immunohistochemistry were as follows: anti-Tyrosine Hydroxylase (diluted 1:1000), anti-Calbindin 
(diluted 1:2000), anti-Parvalbumin (diluted 1:2000), anti-GAD 65/67 (diluted 1:1000), and anti-Calretinin (diluted 1:2000). In addition, two anti-AchE antibodies were tested: a mouse monoclonal antibody (Sigma; clone ZR3, A6970) that has been shown to recognize rodent, feline, and rabbit but not primate AchE (Rackonczay and Brimijoin, 1986); and a rabbit anti-AchE antibody (Sigma; HPA019704) that has been designed to specifically recognize human AchE (see as reference, the human protein atlas, www.proteinatlas.org).

For immunohistochemistry, free-floating sections were rinsed several times in $0.01 \mathrm{M}$ phosphate buffered saline (PBS) and the endogenous peroxidase was blocked by incubating for $30 \mathrm{~min}$ at RT with a solution of 5\% H202 in PBS. After this, sections were rinsed thoroughly in PBS and incubated for $1 \mathrm{~h}$ at RT with $10 \%$ normal serum in PBS containing $0.3 \%$ Triton X-100 (PBS-T). The sections were then incubated for $19 \mathrm{~h}$ at RT with the appropriate primary antibody in a solution containing 3\% normal serum in PBS-T. The following day, the sections were rinsed in PBS and incubated for $45 \mathrm{~min}$ at RT with a secondary antibody [biotinylated horse anti-mouse (Vector Laboratories; BA2001, diluted 1:400) or biotinylated goat anti-rabbit (Vector Laboratories; BA1000, diluted 1:400)] in a solution containing 3\% normal serum in PBS-T. The sections were rinsed in PBS and incubated with an avidin-biotin-complex (Vector Laboratories; PK6100, diluted 1:100) for $45 \mathrm{~min}$ at RT. Finally, the sections were developed using a 3,3'-diaminobenzidine peroxidase kit (Vector Laboratories; SK4100). Negative controls consisting in the omission of the primary antibody and incubation with only $3 \%$ normal serum in PBS-T were performed for all the immunohistochemistry experiments. The sections were mounted on slides, dehydrated, and coverslipped using Eukitt. In addition, GAD65/67 immunolabeled sections were counterstained using thionin prior to coverslipping.

\section{PHOTOGRAPHY AND IMAGE PROCESSING}

Single images or stacks of photographs were obtained on a Nikon Eclipse $50 i$ microscope (Nikon, Tokyo, Japan) connected to a Nikon DS-Fil color digital camera (Nikon). Stacks were only used in the case that the depth of the element to be photographed required this technique to obtain a focused image (e.g., all the Golgi impregnated samples, and in the case of some immunolabeled sections as indicated in the figure legends). Stacks were merged into a single focused image using Heliconfocus software (Heliconsoft, Kharkov, Ukraine). Photomontage and lettering of figure plates were done using CorelDRAW-12 (Corel, Ottawa, Canada).

\section{CELL COUNTS AND MEASUREMENTS}

Cell counts were performed in entire rostrocaudal parallel series labeled for TH ( $n=2$ animals). Each of these series used for cell counts consisted of one out of every six parallel series of $50 \mu \mathrm{m}$ sections collected from each animal. In this scheme, sections used for counts were $300 \mu \mathrm{m}$ apart, which eliminates the possibility of counting twice the same cell. In each section analyzed, all TH labeled cells of the dorsal striatum were counted.

Measurements of cells in TH labeled sections, or cells and processes in Golgi impregnated sections, were performed using the measurement tool of CorelDRAW-12. For soma cross-section size (area) calculations, cell shape was assimilated to the geometrical figure that more closely represented its shape (for example, circle, ellipse, or polygon) and the area was calculated using the mathematical formula for the appropriate geometrical figure.

\section{SCHEMATIC DRAWINGS FOR THE COMPARISON OF THE STRIOSOME/MATRIX ORGANIZATION}

Complete series of sections stained for calbindin, $\mathrm{TH}$, and AchE were used to obtain schematic drawings of the distribution of striosomes in the tree shrew dorsal striatum. The sections were photographed with a $2 \times$ lens and imported into Powerpoint software (Microsoft, Redmond, USA). Contrast was enhanced for a better identification of the striosomes. Drawings were obtained from sections from the most rostral pole of the striatum to the caudal pole of the caudate and putamen. A total of 17 sections per marker were photographed and drawn.

\section{RESULTS \\ SPECIFICITY OF THE ANTIBODIES}

We performed western-blots with protein extracts from whole tree shrew brain and from rat striatum to ensure specificity of the antibodies used in this study. Protein extracts from rat striatum were used as a positive control, since the specificity of the antibodies has already been verified in this species (Bender et al., 2001; Kawaguchi and Hirano, 2002; Meier and Grantyn, 2004; Nafia et al., 2008; Rostkowski et al., 2009). Western-blot experiments showed that the antibodies produced bands that were located at the same level in the gel for both rat striatum and tree shrew brain protein extracts. More importantly, the western-blots also showed that these bands had the proper molecular weight for all the antibodies examined (Figure 1). Acetylcholinesterase antibodies were not assessed by western-blot since this enzyme forms disulfide-linked oligomers with collagenous and lipid-containing structural subunits, which produce an array of different forms with different molecular weights (for a detailed description of acetylcholinesterase forms see www.proteinatlas.org).

\section{NEUROANATOMICAL FEATURES OF THE TREE SHREW STRIATUM}

Based on the study of several thionin-stained series the adult tree shrew striatum was approximately $7.4 \mathrm{~mm}$ long, from its rostral pole to its most caudal level. This represents a $23 \%$ increase in length compared to the adult rat striatum, which is approximately $6 \mathrm{~mm}$ long (based on Paxinos and Watson, 1998). When compared to the human striatum, the length of the tree shrew striatum was approximately $14 \%$ of that of the human [which is approximately $54.4 \mathrm{~mm}$ long (based on Mai et al., 2004)]. The caudate and putamen were visible from the most rostral level of the striatum (Figure 2A), with the putamen extending slightly more caudally than the caudate. The corpus callosum provided a clear dorsal and lateral separation between the striatum and cortex, with the anterior commissure providing a ventral delineation between the dorsal and ventral striatum (Figures 2A,B). The anterior commissure appeared in the most rostral areas of the striatum and continued up to the level in which the GP began to appear in the caudal striatum (Figure 2C). The most defining morphological feature of the tree shrew striatum was the presence of 


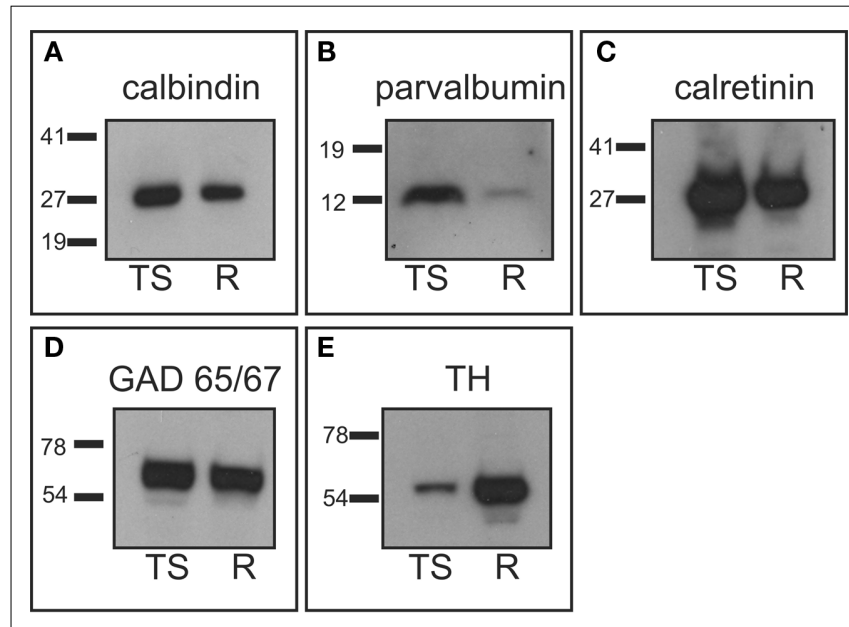

FIGURE 1 | Western-blot analysis. Tree shrew whole brain protein extracts were used to assess antibody specificity, and compared with rat striatal samples used as positive controls. (A) Calbindin western-blot showed a band of approximately $28 \mathrm{kDa}$ in the tree shrew and rat samples. (B) Parvalbumin western-blot presented a single band of approximately $12 \mathrm{kDa}$ in both species, which was more prominent in the tree shrew protein extract. This was likely due to the fact that the tree shrew protein extract also contained cortex tissue, which is known to have a high number of parvalbumin expressing cells (Celio, 1986). (C) The calretinin antibody detected a very strong band of approximately $29 \mathrm{kDa}$ in both species. (D) GAD 65/57 appeared as a very thick band that corresponded with both isoforms of the enzyme. This is due to the fact that this antibody recognizes the two isoforms of GAD and the resolution of the gel did not allow for the separation of the two isoforms. (E) TH appeared as a single band of approximately $60 \mathrm{kDa}$ that was stronger in the rat sample. This difference was likely due to the fact that the rat sample contained only striatum, which has a very high concentration of $\mathrm{TH}$, while the tree shrew sample was a whole brain protein extract. R, rat; TS, tree shrew. Numbers indicate the molecular weight in kilodaltons.

a well defined and developed internal capsule, which created a clear separation between the caudate nucleus and the putamen (Figures 2A-C).

\section{LABELING FOR PARVALBUMIN}

Throughout the rostrocaudal extent of the striatum the general pattern of staining did not differ between the caudate nucleus and the putamen, although within each nucleus there were qualitative differences in the staining for parvalbumin (Figures $3 \mathrm{~A}-\mathrm{C}$ ). In both nuclei, areas of higher and lower staining for parvalbumin immunoreactivity (parv-ir) were clearly observed from the most rostral to the most caudal regions (Figures 3D-F). This labeling pattern was due to differences in the density of parv-ir processes, with a lower density of processes present in the low parv-ir areas (Figures 3D-F). This pattern of expression partially matched a striosome/matrix distribution in rostral areas, with lower parv-ir in the striosomes. Parvalbuminpositive cells were loosely clustered with most of these clusters avoiding the low parv-ir areas, although some parv-ir cells were located within them (Figures 3D-F). These parvalbumin neurons also presented two to five labeled processes per neuron (Figures 3D-F). Parv-ir cells with different patterns of staining (i.e., from light to very dark cytoplasm labeling) appeared

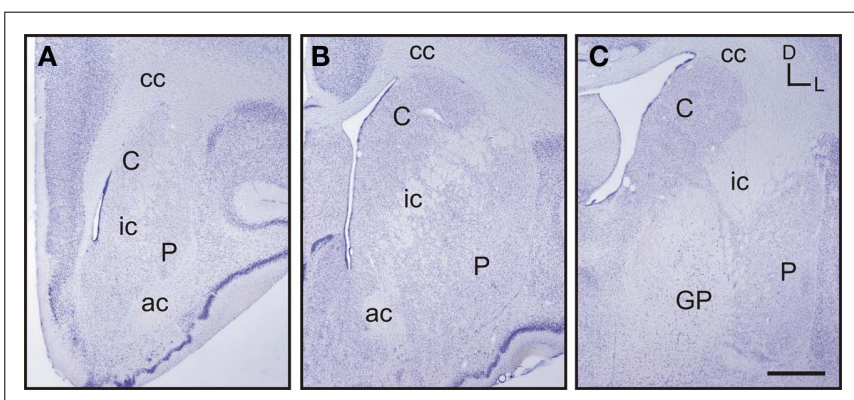

FIGURE 2 | Images of representative Nissl-stained rostrocaudal sections throughout the tree shrew striatum. (A) Rostral striatum. Note the clear separation of the caudate $(C)$ and putamen $(P)$ by the internal capsule (ic). (B) Mid striatum. The internal capsule (ic) is still present. The corpus callosum (cc) and anterior commissure (ac) are well formed and delineate the extent of the striatum. (C) Caudal striatum. The caudate (C) and putamen (P) are separated by the internal capsule (ic). The anterior commissure is no longer visible and the globus pallidus (GP) is now present. Coordinates indicate the orientation of the sections in the figure (D, dorsal; L, lateral). Scale bars: $1 \mathrm{~mm}$ in (A-C).

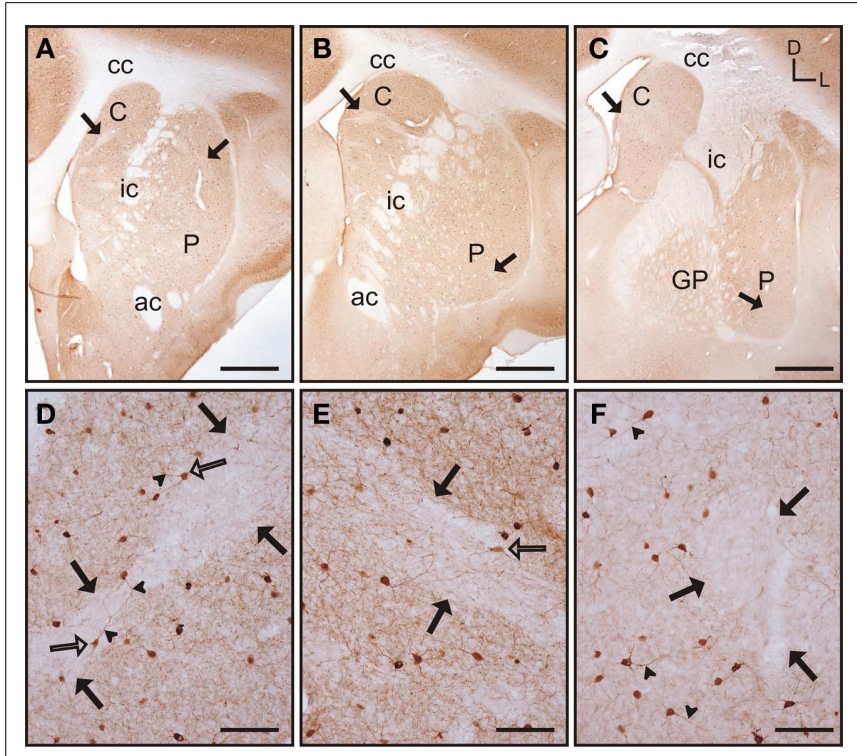

FIGURE 3 | Parvalbumin immunostaining in the tree shrew striatum. (A-C) Low magnification images of the rostral (A), mid (B), and caudal (C) striatum, showing the presence of bands of low labeling (black arrows) in these three rostrocaudal regions. (D) Detail of a low parv-ir band in the rostral caudate (black arrows). Several labeled neurons present multiple slender and long labeled processes (arrowheads), crossing in some cases from rich immunolabeled areas into the low parv-ir band. (E) Detail of parvalbumin labeling in the mid caudate showing the presence of a low immunoreactive band (black arrows) flanked by parv-ir cells distributed in clusters. Some labeled neurons are located in the low labeling band (open arrow). (F) Detail of parv-ir cells in the caudal putamen showing immunolabeled processes (arrowheads). A low immunolabeling band is located on the right and is devoid of labeled cells (black arrows). Note also in (D-F) the abundance of labeled processes in the parvalbumin rich areas, but also the presence of some processes in the areas with low parv-ir. Figures (D-F) are composite images of several Z-stack images. Coordinates indicate the orientation of the sections in the figure ( $D$, dorsal; $L$, lateral). ac, anterior commissure; $C$, caudate; cc, corpus callosum; ic, internal capsule; GP, globus pallidus; , putamen. Scale bars: $1 \mathrm{~mm}$ in (A-C); $100 \mu \mathrm{m}$ in (D-F). 
distributed together in the same area (Figures 3D-F). Finally, parv-ir was absent from both the internal capsule and the corpus callosum.

\section{LABELING FOR CALRETININ}

In the tree shrew striatum there were clear differences in the general pattern of staining for calretinin between the caudate and putamen. In the caudate nucleus calretinin immunoreactivity (calretir) remained constant throughout its rostrocaudal extent, without any patterns or bands of different labeling (Figures 4A-C). Rostral areas of the putamen had a similar pattern of staining to the caudate; while in mid and caudal areas calret-ir was qualitatively lower than in the caudate (Figures $4 \mathrm{~A}-\mathrm{C}$ ). This difference was mainly due to the presence of an area of low calret-ir that became progressively larger in more caudal regions. In rostral regions of the putamen the low calret-ir area occupied a small part of the nucleus and was restricted to a dorsolateral position, while in mid regions this low calret-ir area extended into ventrolateral positions (Figure 4B). In caudal regions the low calret-ir area encompassed the majority of the putamen, from dorsolateral to lateral and medial positions (Figure 4C). In rostral areas of the caudate and putamen calret-ir neurons were scarce. This pattern continued throughout the rostrocaudal extent of the caudate (Figures 4D,F), while in mid regions of the putamen

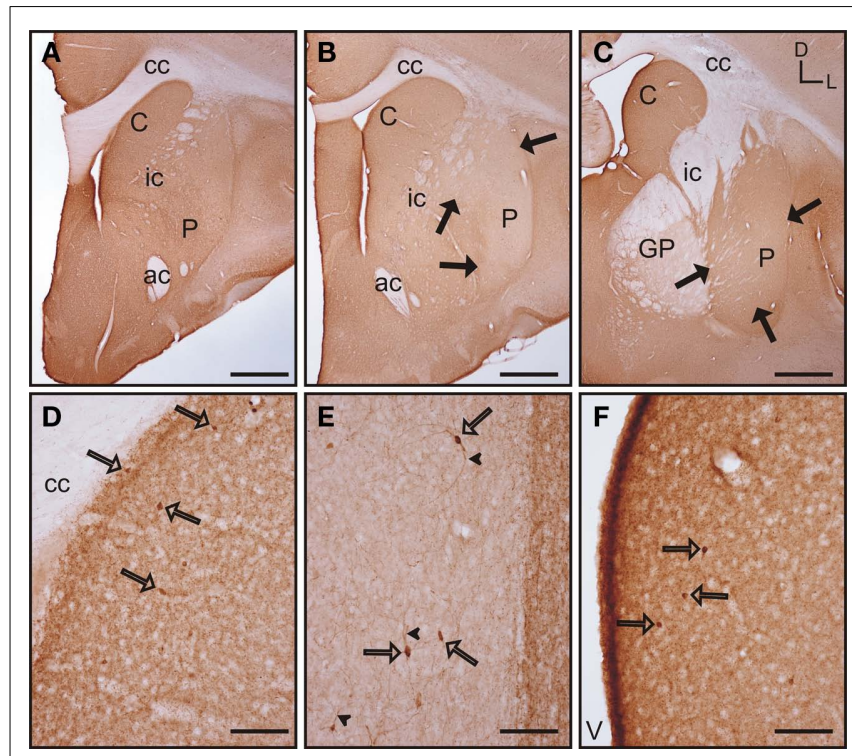

FIGURE 4 | Calretinin immunoreactivity in the tree shrew striatum. (A-C) Low magnification images of calretinin labeling in the rostral (A), mid (B), and caudal (C), striatum. Note the presence of a large low calret-ir band in the mid and caudal regions of the putamen [black arrows in $\mathbf{( B , C )}$ ]. (D) Detail of calretinin labeling in the rostral caudate showing scattered calret-ir cells (open arrows) embedded in a calretinin rich neuropil. (E) High magnification image of the mid putamen showing several calret-ir neurons (open arrows) located in an area of low calret-ir neuropil. Note also the presence of labeled processes in these cells (arrowheads). (F) Calret-ir cells in the caudal region of the caudate (open arrows). Coordinates indicate the orientation of the sections in the figure ( $D$, dorsal; $L$, lateral). ac, anterior commissure; C, caudate; cc, corpus callosum; ic, internal capsule; GP, globus pallidus; $P$, putamen; $V$, ventricle. Scale bars: $1 \mathrm{~mm}$ in (A-C); $100 \mu \mathrm{m}$ in (D-F). labeled neurons were more frequently observed (Figure 4E) and became scarce again in caudal areas. In the caudate calret-ir neurons were mainly relegated to dorsolateral areas of the nucleus (Figures 4D,F). In the putamen positive neurons were mainly located in the low calret-ir area (Figure 4E), with neurons located primarily in dorsolateral positions in rostral regions, and progressing into more ventral and ventromedial positions caudally. Both nuclei contained moderately stained neurons, with labeling located in the cytoplasm and the initial segment of one or two processes (Figures 4D-F). In addition, calret-ir processes were observed crossing the internal capsule but not the anterior commissure.

\section{LABELING FOR CALBINDIN}

In the rostral and mid areas of the striatum the caudate and putamen presented a similar pattern of calbindin immunoreactivity (calb-ir; Figures 5A-B), while in more caudal areas the caudate nucleus displayed more intense staining than the putamen (Figure 5C). Starting at the most rostral level of the striatum there were areas with qualitative differences in calbindin labeling within both the caudate and putamen. These areas followed a classical striosome/matrix organization, with stronger calb-ir present in the

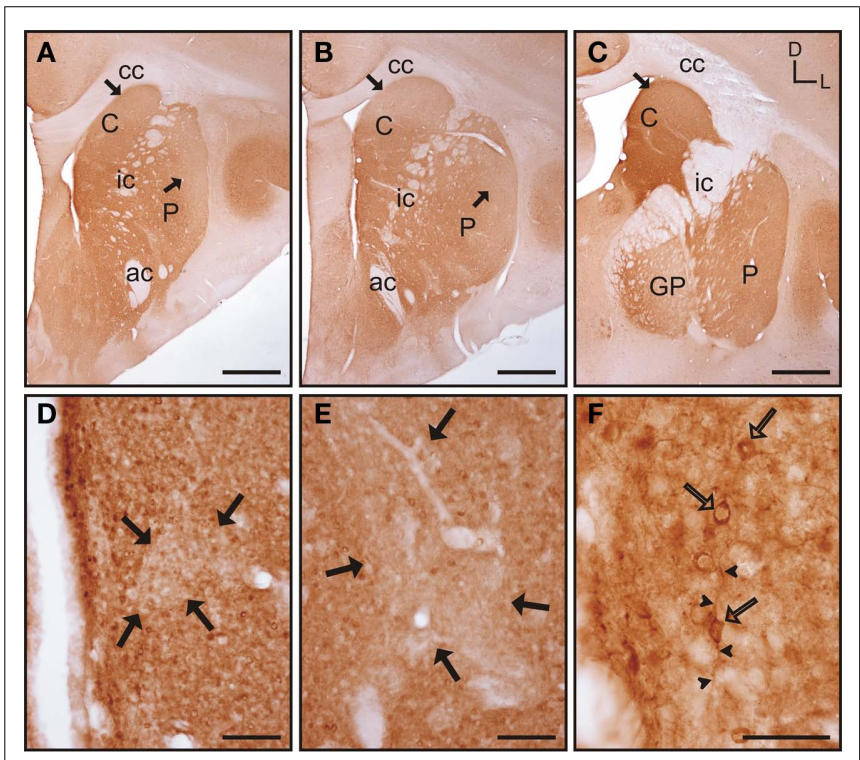

FIGURE 5 | Calbindin immunolabeling in the tree shrew striatum. (A-C) Low magnification images of the rostral (A), mid (B), and caudal (C) tree shrew striatum immunolabeled for calbindin. Note the presence of areas with higher and lower levels (black arrows) of calbindin immunolabeling. Also note the difference of labeling between the caudate and putamen in caudal areas (C). (D) Detail of calbindin labeling demonstrating the presence of striosomes in the caudate (black arrows). (E) Detail of the putamen in which the striosome/matrix organization is also present. Note that both in the caudate (D) and in the putamen (E) the striosomes (black arrows) are not completely devoid of immunolabeling. (F) Detail of calbindin labeled cells (open arrows) in the caudate of the tree shrew. These cells contain cytoplasmic staining as well as labeling in the initial segment of processes (arrowheads). Coordinates indicate the orientation of the sections in the figure ( $D$, dorsal; $L$, lateral). ac, anterior commissure; $C$, caudate; cc, corpus callosum; ic, internal capsule; GP, globus pallidus; $P$, putamen. Scale bars: $1 \mathrm{~mm}$ in (A-C); $100 \mu \mathrm{m}$ in (D-E); $50 \mu \mathrm{m}$ in (F). 
matrix than in the striosomes (Figures 5A-E). At the most rostral level of the striatum two large striosomes with lower calb-ir were observed; one in the dorsomedial caudate, and another in the dorsolateral putamen. In addition, smaller low calb-ir striosomes were present in more ventral areas of both nuclei (Figure 5A). In mid areas, the large dorsolateral striosome of the putamen and the dorsomedial striosome of the caudate were still present (Figure 5B). In caudal areas, the dorsomedial striosome of the caudate was still visible, while in the putamen the striosome/matrix pattern was more difficult to discern due to the fact that most of the putamen was occupied by a large striosome (Figure 5C). A more detailed observation of the striosome and matrix compartments revealed that the matrix clearly presented a more abundant population of calb-ir cells, but cellular staining was also present in the striosomes (Figures 5D-E). Neurons located within the striosomes were generally lighter in their staining than the ones in the matrix. At the cellular level, calb-ir appeared as cytoplasmic labeling and in some cases the initial segment of one or two processes was also labeled (Figure 5F).

\section{LABELING FOR TYROSINE HYDROXYLASE}

The caudate and putamen contained abundant TH immunoreactivity (TH-ir) and the pattern of staining was similar between the two nuclei. Also, there were no differences in the pattern of staining along the rostrocaudal extent of each of these nuclei (Figures 6A-C). However, a patch-like pattern of labeling for THir was observed in both nuclei, with the striosomes presenting lower TH-ir. Even when this pattern was present at all rostrocaudal levels of the caudate and putamen, the patch-like areas were more prominent in rostral and mid regions, especially in the caudate (Figures 6A,B). At high magnification this pattern of immunolabeling corresponded with areas that contained high and low density of TH-ir processes and terminals (Figures 6D-E). The corpus callosum, internal capsule, and the anterior commissure also contained a small number of $\mathrm{TH}$-ir processes. A small number of $\mathrm{TH}$-ir cells were present in the tree shrew dorsal striatum. These cells were not homogeneous in morphology, and according to their size they could be classified in three subtypes (Figures $6 \mathrm{~F}-\mathbf{H}$ ). A summary of the morphological features and relative abundance of each type of TH-ir cells is shown in Table 1.

We found a great variability in the total number of TH-ir cells between the two animals used for neuronal counts [animal \#1 had $175 \mathrm{TH}$-ir cells/series which yields a total of $1050 \mathrm{TH}$-ir cells for the whole dorsal striatum; animal \#2 had $291 \mathrm{TH}$-ir cells/series which yields a total of $1746 \mathrm{TH}$-ir cells]. In contrast with the high variability observed in the total number of TH-ir cells, the percentage of cells of each subtype was very similar for both animals (see the low SD in Table 1).

\section{LABELING FOR ACETYLCHOLINESTERASE}

Acetylcholinesterase (AchE) histochemistry was classically used as one of the first staining techniques that revealed the striosome and matrix compartments (Graybiel and Ragsdale, 1978). This technique has as a drawback in the need for special fixatives (see Karnovsky and Roots, 1964), or very lightly fixed tissue (see, e.g., Puelles et al., 1996). This is not compatible with the immunohistochemical detection of other markers for striosome/matrix

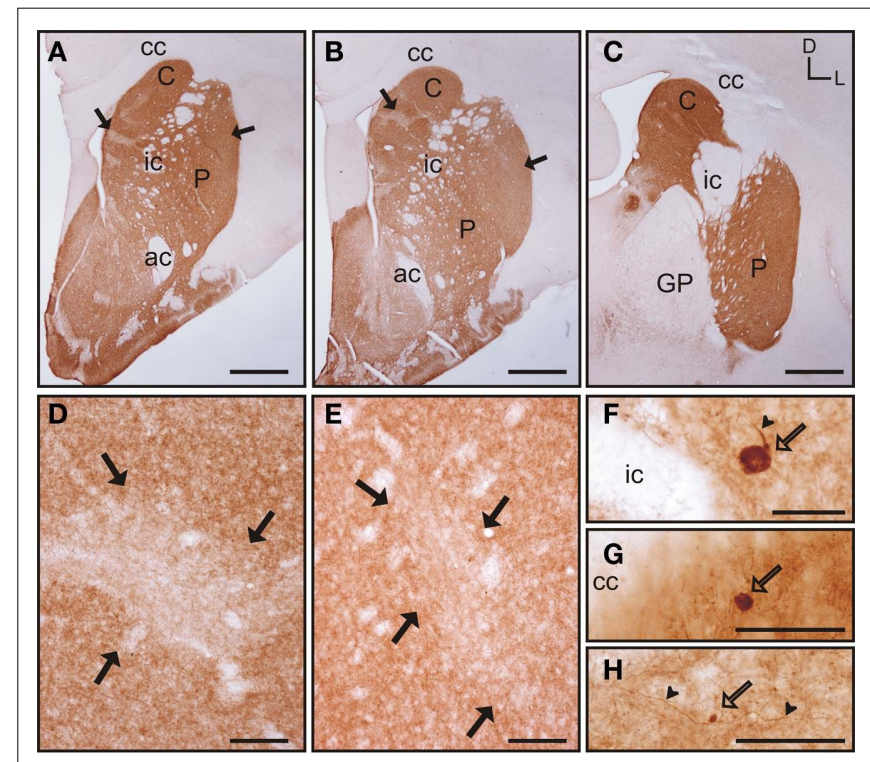

FIGURE 6 | Tyrosine hydroxylase immunostaining in the tree shrew striatum. (A-C) Low magnification images of TH labeling in the rostral (A), mid (B), and caudal (C) striatum, showing a patch-like pattern in both the caudate and the putamen. This pattern is more prominent in rostral and medial regions (A,B). (D) Detail of a low TH-ir striosome (black arrows) in the mid caudate. (E) Detail of a low TH-ir striosome at the level of the mid putamen (black arrows). (F-H) Detail images of large (F), medium-sized (G), and very small $\mathbf{( H )}$ TH labeled cells in the tree shrew dorsal striatum. Some

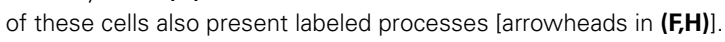
Coordinates indicate the orientation of the sections in the figure $(D$, dorsal; $\mathrm{L}$, lateral). ac, anterior commissure; C, caudate; cc, corpus callosum; ic, internal capsule; GP, globus pallidus; P, putamen. Scale bars: $1 \mathrm{~mm}$ in (A-C); $100 \mu \mathrm{m}$ in (D-E); $50 \mu \mathrm{m}$ in (F-H).

compartments (e.g., calbindin, $\mathrm{TH}$ ) in parallel series from the same animal. To avoid this problem, instead of the histochemical procedure for the detection of AchE, we used immunohistochemistry to detect this enzyme. Two anti-acetylcholinesterase antibodies were used in our study: the first one has been shown to specifically recognize AchE in rat, feline and rabbit while not recognizing AchE in mouse, human, and chicken (Rackonczay and Brimijoin, 1986; see also Material and Methods). This antibody did not produce any labeling in the tree shrew striatum while clearly recognizing AchE in adult rat striatal sections used as a positive control (data not shown). The second antibody tested has been previously demonstrated to specifically recognize AchE in human brain (see Material and Methods). This second antibody produced positive labeling in the tree shrew striatum (Figure 7), while not producing any labeling in the rat striatum (data not shown).

In the tree shrew striatum AchE immunoreactivity (AchE-ir) displayed a "patchy distribution" with light striosomes and darker matrix. This pattern was evident in rostral areas of the dorsal striatum and became increasingly difficult to discern in mid and caudal regions (Figures 7A-C). At higher magnification it was evident that the striosomes contained a lower density of AchE-ir processes (Figure 7D). AchE-ir cells were present in all rostrocaudal areas of the caudate and putamen. These AchE-ir cells were moderately labeled cells and often presented labeled processes (Figures 7E-F). 
Table 1 | Morphological features of TH labeled neurons in the tree shrew striatum.

\begin{tabular}{|c|c|c|c|}
\hline Cell type & $\begin{array}{l}\text { Average size } \\
\text { (cross-section; } \mu \mathrm{m}^{2} \text { ) }\end{array}$ & Shape, processes, and staining & $\begin{array}{l}\text { Mean percentage of } \\
\text { total TH-ir cells }(\%)\end{array}$ \\
\hline Very small & $20.47 \pm 9.92$ & Oval to polygonal. Several thin processes. Staining moderate, rarely granular. & $69.6 \pm 3.11$ \\
\hline Small to medium & $73.12 \pm 17.66$ & $\begin{array}{l}\text { Round to polygonal with one process frequently observed. Rarely several } \\
\text { processes. Staining from strong smooth to granular. }\end{array}$ & $29.11 \pm 3.27$ \\
\hline Large to very large & $259.63 \pm 161.95$ & Round to oval with one process visible. Smooth strong labeling. & $1.25 \pm 0.16$ \\
\hline
\end{tabular}

TH immunoreactivity cells in the dorsal striatum were counted and classified in a total of two complete series of striatal sections from two different adult male tree shrews. Each series was composed of sections $300 \mu \mathrm{m}$ apart and a total of 17 sections were studied per animal.
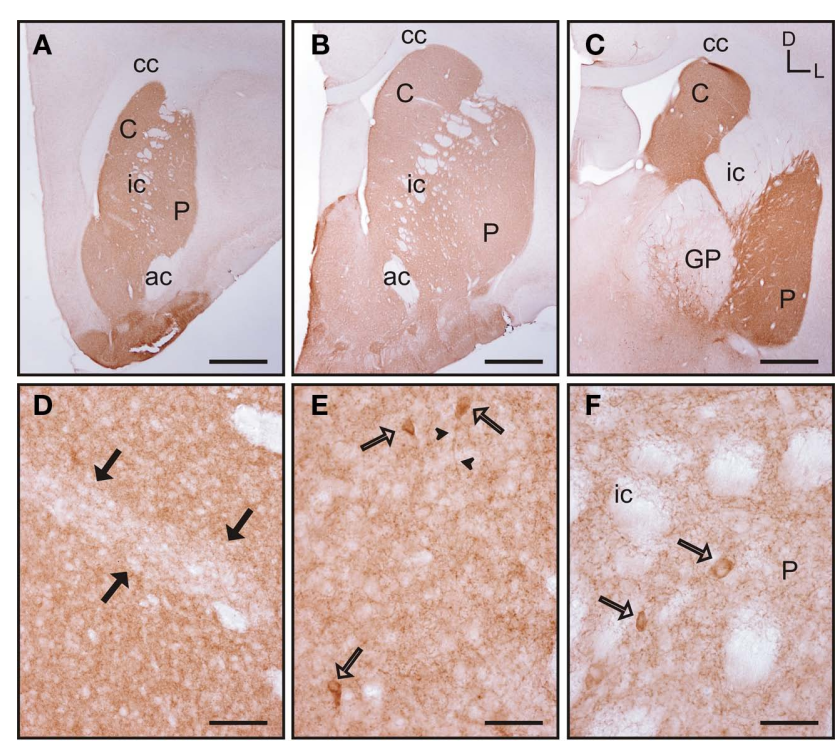

FIGURE 7 | Acetylcholinesterase immunolabeling in the tree shrew striatum. (A-C) Low magnification images of AchE labeling in the rostral (A), mid (B), and caudal (C) striatum showing a patchy distribution of immunostaining. (D) Detail of a patch of low AchE-ir (black arrows) in the rostral caudate. (E,F) Detail of AchE labeled cells (open arrows) in the caudate (E) and putamen (F). Some of these cells present labeling in the initial segment of a process [arrowheads in (E)]. Coordinates indicate the orientation of the sections in the figure ( $D$, dorsal; $L$, lateral). ac, anterior commissure; C, caudate; CC, corpus callosum; ic, internal capsule; GP, globus pallidus; $P$, putamen. Scale bars: $1 \mathrm{~mm}$ in (A-C); $100 \mu \mathrm{m}$ in (D); $50 \mu \mathrm{m}$ in (E-F)

These AchE-ir cells appeared mostly in the matrix, sometimes surrounding the borders of the striosomes (Figures 7E-F).

\section{COMPARISON OF THE STRIOSOME/MATRIX ORGANIZATION REVEALED BY CALBINDIN, TH, AND AchE IMMUNOHISTOCHEMISTRY}

Schematic drawings of parallel sections were obtained for the three markers to analyze the pattern of striosome/matrix distribution (Figures 8-10). Calbindin and $\mathrm{TH}$ yielded the best definition of the striosome and matrix compartments. In rostral to mid regions calbindin demonstrated the presence of a large dorsolateral patch in the caudate that was still present in mid and caudal regions (Figures 8A, 9A, and 10A). In TH-ir and AchE-ir sections, one or several closely distributed striosomes also appeared in this dorsolateral area of the caudate across the rostrocaudal extent of this
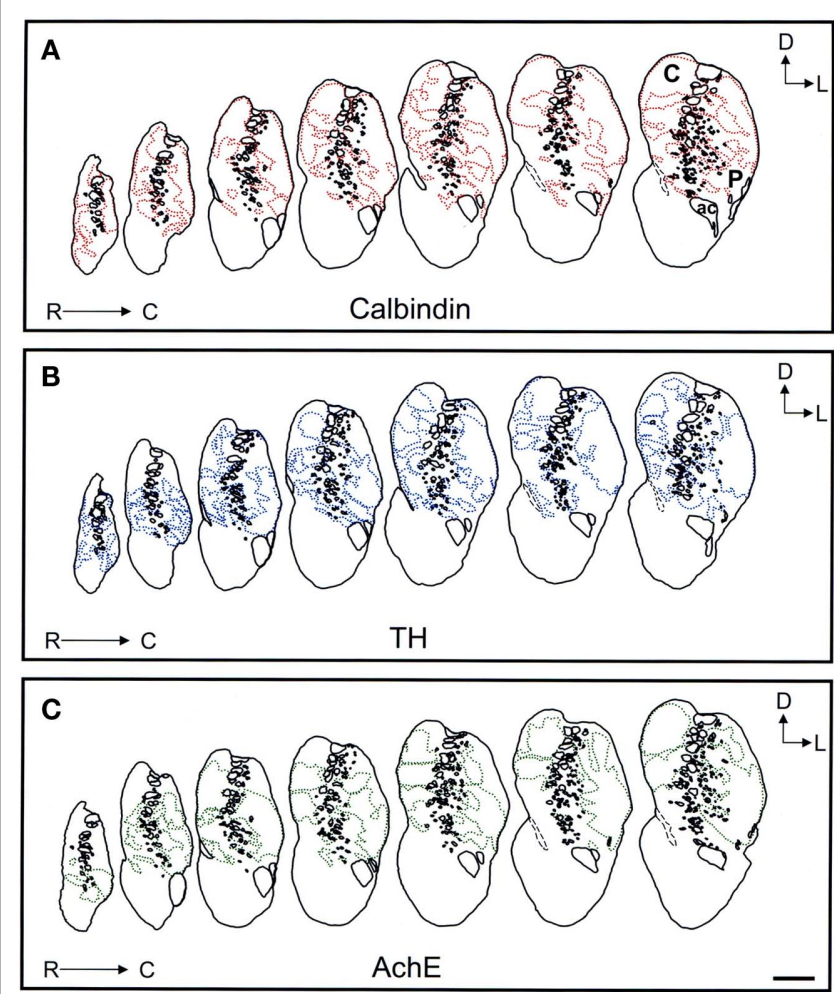

FIGURE 8 | Schematic drawings of the rostral to mid areas of the tree shrew dorsal striatum. These line drawings show the patch (striosomes) distribution for calbindin (A), TH (B), and AchE (C). Drawings were obtained from whole series of the same animal stained for the different markers. Each of the contiguous rostrocaudal sections of the same marker are separated $300 \mu \mathrm{m}$. Separation between parallel sections labeled for calbindin or $\mathrm{TH}$ is $50 \mu \mathrm{m}$, while between calbindin and AchE is $100 \mu \mathrm{m}$. Coordinates indicate the orientation of the sections in the figure $(D$, dorsal; $\mathrm{L}$, lateral). ac, anterior commissure; C, caudate; $\mathrm{P}$, putamen. Scale bars: $1 \mathrm{~mm}$ in $\mathbf{( A - C )}$

nucleus (Figures $\mathbf{8 B}, \mathbf{C}, \mathbf{9 B}, \mathbf{C}$, and $\mathbf{1 0 B}, \mathbf{C}$ ). However, there were some slight differences mainly in the most rostral areas of the caudate (for example, the dorsolateral striosome appeared earlier in the calb-ir sections than in the case of sections labeled for TH or AchE; Figures 8A-C). The three markers consistently revealed the presence of smaller striosomes in more ventral areas of the caudate, but the pattern of striosome/matrix distribution was more closely matched for calbindin and TH than for AchE (Figures 8-10). 

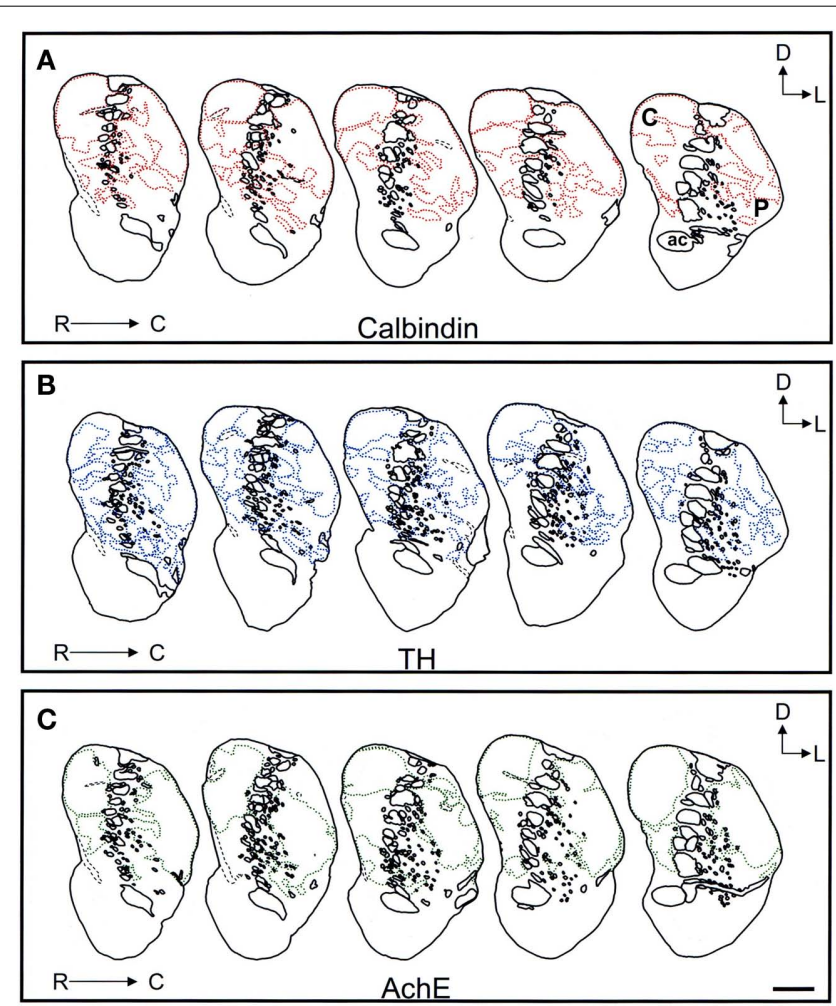

FIGURE 9 | Schematic drawings of the mid to caudal areas of the tree shrew dorsal striatum. These line drawings show the patch (striosomes) distribution for calbindin (A), TH (B), and AchE (C). Drawings were obtained from whole series of the same animal stained for the different markers. Each of the contiguous rostrocaudal sections of the same marker are separated $300 \mu \mathrm{m}$. Separation between parallel sections labeled for calbindin or $\mathrm{TH}$ is $50 \mu \mathrm{m}$, while between calbindin and AchE is $100 \mu \mathrm{m}$. Coordinates indicate the orientation of the sections in the figure $(D$, dorsal; $\mathrm{L}$, lateral). ac, anterior commissure; $\mathrm{C}$, caudate; $\mathrm{P}$, putamen. Scale bars: $1 \mathrm{~mm}$ in $\mathbf{( A - C )}$.

In the putamen the three markers showed a very consistent pattern with a large striosome (sometimes observed as several closely attached striosomes in rostral regions) that occupied most of the nucleus. This large striosome was especially consistent in mid and caudal regions (Figures 8-10).

\section{LABELING FOR GAD65/67 AND MORPHOLOGY OF MEDIUM SPINY NEURONS}

The caudate and putamen presented a similar pattern of immunolabeling for GAD 65/67, and this remained constant throughout the rostrocaudal extent of both nuclei (Figures 11A-C). GAD $65 / 67$ staining within the caudate and putamen was observed as strongly labeled puncta surrounding unstained neurons. The corpus callosum and the anterior commissure also had GAD 65/67 labeling. Staining within these fiber bundles was observed as scattered immunoreactive processes, running in parallel to the fiber bundles (Figure 11D). In addition, the corpus callosum also contained a scarce number of GAD 65/67 positive neurons (Figure 11D). In the internal capsule immunoreactive processes were observed crossing perpendicular to the fiber
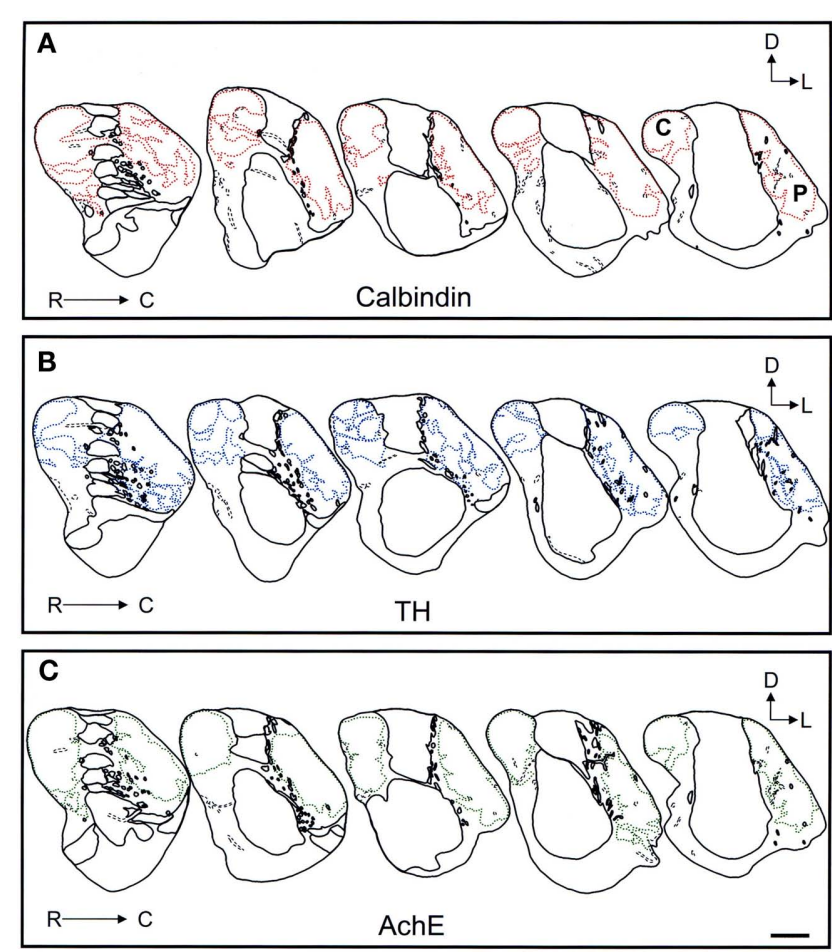

FIGURE 10 | Schematic drawings of the most caudal areas of the tree shrew dorsal striatum. These line drawings show the patch (striosomes) distribution for calbindin (A), TH (B), and AchE (C). Drawings were obtained from whole series of the same animal stained for the different markers. Each of the contiguous rostrocaudal sections of the same marker are separated $300 \mu \mathrm{m}$. Separation between parallel sections labeled for calbindin or $\mathrm{TH}$ is $50 \mu \mathrm{m}$, while between calbindin and AchE is $100 \mu \mathrm{m}$. Coordinates indicate the orientation of the sections in the figure $(D$, dorsal; L, lateral). C, caudate; $P$, putamen. Scale bars: $1 \mathrm{~mm}$ in (A-C).

bundles (Figure 11E). Due to the strong labeling of processes and terminals, the detection of immunopositive neurons in the caudate and putamen was difficult. However, the use of Nissl counterstained sections allowed discernment of the presence of some GAD65/67 labeled neurons that mostly contained cytoplasmic granular labeling (Figure 11F).

Golgi impregnation was used to study in more detail the morphological features of the medium spiny neurons of the tree shrew striatum. A summary of their morphological features is shown in Table 2. This type of GABAergic neurons were easily distinguished from other cell types in Golgi impregnated tissue (Figures 12A-C). A qualitative observation revealed that these cells presented a lower density of dendritic spines in their primary dendrites than in their secondary and higher order dendrites (Figures 12B,C), which were densely covered with dendritic spines of diverse morphologies (Figures 12D,E).

\section{DISCUSSION}

Recent advances in molecular phylogenetics have allowed the study of large DNA data sets and reach the current consensus that Dermoptera (flying lemurs) and Scandentia (tree shrews) are the closest living relatives to Primates (Adkins and Honeycutt, 1991; 
Table 2 | Morphological features of the medium spiny neurons of the tree shrew striatum.

\begin{tabular}{lllll}
\hline Shape of soma & $\begin{array}{l}\text { Number of } \\
\text { primary dendrites }\end{array}$ & $\begin{array}{l}\text { Mean soma } \\
\text { cross-section (area; } \boldsymbol{\mu \mathbf { m } ^ { 2 } )}\end{array}$ & $\begin{array}{l}\text { Mean distance soma to } \\
\text { secondary dendrites }(\boldsymbol{\mu} \mathbf{m})\end{array}$ & $\begin{array}{l}\text { Mean distance soma to } \\
\text { first dendritic spine }(\boldsymbol{\mu} \mathbf{m})\end{array}$ \\
\hline Round to polygonal & $5-6$ & $273.26 \pm 49.86$ & $15.86 \pm 6.19$ & $10.36 \pm 6.69$ \\
\hline
\end{tabular}

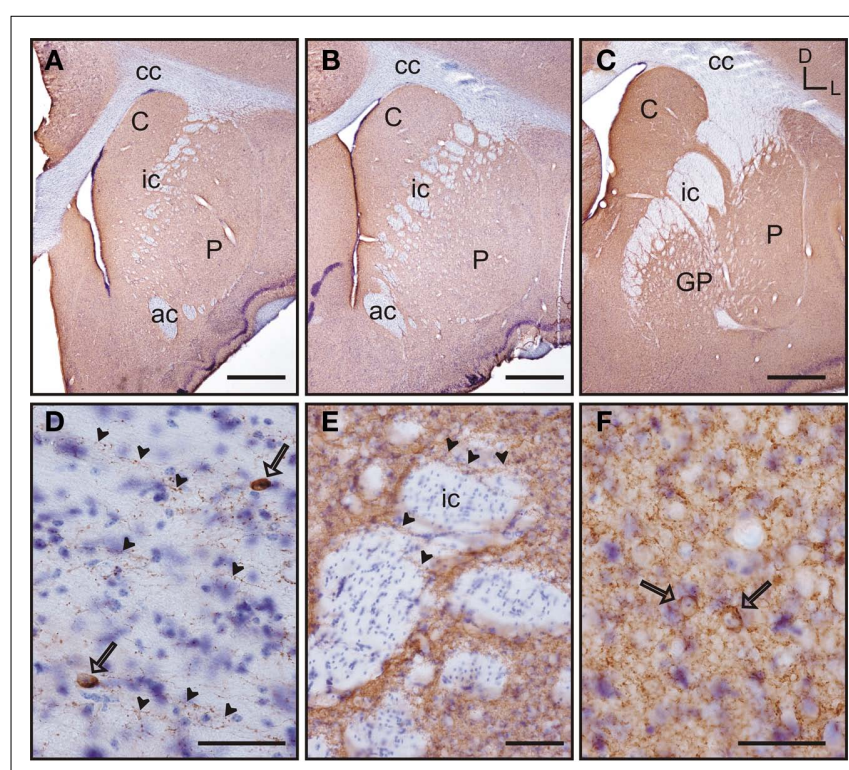

FIGURE 11 | GAD 65/67 immunolabeling in the tree shrew striatum. GAD65/67 stained sections were counterstained with thionin (Nissl stain) to improve the visualization of labeled neurons. (A-C) Low magnification images of GAD 65/67 labeling in the rostral (A), mid (B), and caudal (C) striatum. GAD 65/67 appears as a rather homogeneous labeling. (D) Detail of GAD 65/67 immunoreactive fibers (arrowheads) running in parallel to the fiber bundles of the corpus callosum. Some GAD 65/67 positive neurons are also observed (open arrows). (E) Detail of GAD 65/67 immunolabeled fibers (arrowheads) crossing perpendicularly to the fiber bundles of the internal capsule (ic). (F) Detail of two GAD 65/67 immunoreactive neurons (open arrows) embedded in a rich GAD 65/67-ir neuropil in the caudate nucleus. Coordinates indicate the orientation of the sections in the figure (D, dorsal; L, lateral). ac, anterior commissure; C, caudate; cc, corpus callosum; ic, internal capsule; GP, globus pallidus; P, putamen. Scale bars: $1 \mathrm{~mm}$ in $\mathbf{( A - C ) ; ~} 100 \mu \mathrm{m}$ in (E); $50 \mu \mathrm{m}$ in (D,F).

Liu et al., 2001; Murphy et al., 2001; Janecka et al., 2007). Even when these molecular studies have advanced our knowledge on tree shrew phylogenetics, data in other related fields such as comparative neuroanatomy is fragmented and largely focused on the study of the visual system. Comprehensive studies on the visual system together with studies of cortical territories have already provided hints that indicate the tree shrew brain may share many common features with primates (see, e.g., Lyon et al., 2003; Remple et al., 2007; Chomsung et al., 2010). A second important set of data comes from behavioral and physiological studies that provide compelling evidence that tree shrews are closer to primates in their responses than to rodents (Bartolomucci et al., 2001; see as reviews Fuchs and Flugge, 2002; Fuchs, 2005). All this body of data strongly supports that tree shrews are closely related to primates in brain anatomy and function, and can be a suitable model for neuropathology studies (see as a review Cao et al., 2003).

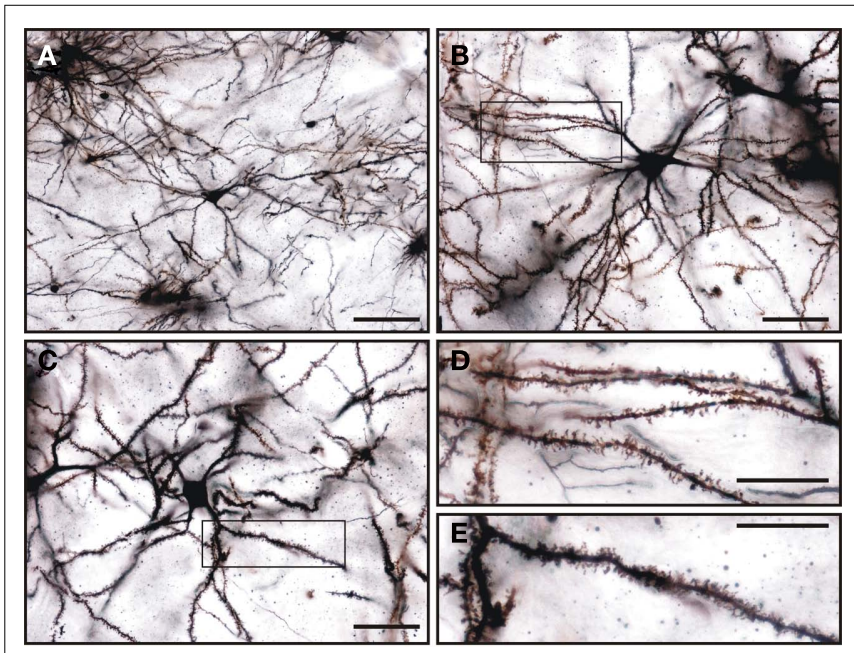

FIGURE 12 | Golgi impregnated medium spiny neurons in the tree shrew dorsal striatum. (A-C) General view of medium spiny neurons of the tree shrew striatum showing the classical morphology of this type of neuron. Details of dendrites and dendritic spines are shown in (D) for the boxed area shown in (B), and in (E) for the boxed area shown in (C). Note the presence of a high density of dendritic spines of different morphologies in these dendrites. Scale bars: $100 \mu \mathrm{m}$ in (A); $50 \mu \mathrm{m}$ in (B-C); $25 \mu \mathrm{m}$ in (D-E).

The tree shrew striatum has not been previously studied in detail. Although some research has provided punctual data concerning this brain region (Lin et al., 1984; Mijnster et al., 1999; Isovich et al., 2000; Day-Brown et al., 2010), as far as we are aware this is the first study that aims to characterize this brain area in detail. The tree shrew striatum presents most of the main morphological features observed in primates and other higher mammals, such as the presence of a well-developed internal capsule that clearly separates the caudate and the putamen nuclei. These features place the tree shrew striatum, at least from a morphological perspective, closer to primates than to rodents. Based on these morphological similarities, we undertook the study of several wellknown striatal markers in order to further characterize this brain region.

The pattern of expression of some of the major calciumbinding proteins has been thoroughly studied in the striatum of mammals including primates and rodents (Cote et al., 1991; Hiroi, 1995; Figueredo-Cardenas et al., 1996; Parent et al., 1996; Holt et al., 1997; Prensa et al., 1998; Wu and Parent, 2000; Morel et al., 2002). Some of these proteins differ considerably in their pattern of distribution in the striatum when primates and rodents are compared (De las Heras et al., 1994; Wu and Parent, 2000). Among these calcium-binding proteins, calbindin has been proven to reveal the striosome/matrix cytoarchitecture of the dorsal striatum 
(see, e.g., Liu and Graybiel, 1992; Kubota and Kawaguchi, 1993; Rajakumar et al., 1993; Parent et al., 1996; Holt et al., 1997; Morel et al., 2002).

Labeling for parvalbumin in the tree shrew striatum has been recently reported by Day-Brown et al. (2010) as a marker for the striosome/matrix compartments. These authors showed that parvalbumin labeling was low in the striosomes and high in the matrix, in a similar manner to what we found in our study for rostral areas of the caudate and putamen (present results). However, our detailed study of all the rostrocaudal extent of the striatum did not support that parvalbumin aligns with a striosome/matrix organization in caudal areas. This discrepancy may be due to the fact that these authors studied the expression of parvalbumin in specific areas of the striatum, while our study encompasses the analysis of the entire rostrocaudal extent of this region. In accordance with our data, in primates including humans, bands of higher and lower staining have also been found in the caudate and putamen (Waldvogel and Faull, 1993; Parent et al., 1996; Holt et al., 1997; Prensa et al., 1999), but these bands did not completely align with the striosome/matrix organization revealed by calbindin staining (Waldvogel and Faull, 1993; Parent et al., 1996). Apart from the partial recognition of the striosome/matrix pattern some authors have reported a rostrocaudal gradient of labeling for parv-ir in the squirrel monkey and in human striatum (Parent et al., 1996; Prensa et al., 1999). However, Holt et al. (1997) and Bernacer et al. (2008) did not report the existence of a rostrocaudal gradient for parvalbumin in the human striatum. In our study we did not find any rostrocaudal gradient in the tree shrew caudate and putamen. An additional increasing dorsoventral gradient has been reported in human and non-human primates (Parent et al., 1996; Holt et al., 1997; Prensa et al., 1999; Morel et al., 2002; Bernacer et al., 2008). This gradient was not observed in the tree shrew (present results).

Parv-ir neurons in the tree shrew striatum appeared mostly distributed in the matrix and were loosely arranged in clusters that may correspond with the so-called matrisomes (clusters of projection neurons) found in the striatal matrix in felines and primates (Gimenez-Amaya and Graybiel, 1990; Selemon and GoldmanRakic, 1990; Graybiel et al., 1991; Desban et al., 1995). Parv-ir cells in the tree shrew dorsal striatum were morphologically very similar to these cells in human and non-human primates, presenting cytoplasmic labeling with several processes emanating from the stained cell (Holt et al., 1997; Prensa et al., 1999; Wu and Parent, 2000; Kataoka et al., 2010). In addition, as reported in humans (Prensa et al., 1999), parv-ir cells were distributed both in areas of high and low parv-ir. In summary, despite of the lack of a dorsoventral gradient, parvalbumin expression in the tree shrew dorsal striatum presents many of the same core features of parv-ir in human and non-human primates.

Immunolabeling for calretinin in the tree shrew striatum presented both similarities and differences when compared to primates. In the tree shrew putamen there was a decreasing rostrocaudal gradient of calretinin labeling, largely due to the presence of a band of low immunoreactivity in the dorsal region. This low immunoreactivity band increased in size in more caudal regions of the striatum, occupying more ventrolateral and medial positions within the putamen. A previous study reported a similar pattern of low calret-ir in dorsal areas of the squirrel monkey striatum, although in that case both the putamen and caudate presented this gradient (Parent et al., 1996). Calretinin positive neurons in the tree shrew dorsal striatum were characterized by the presence of one to two labeled processes, and were medium-sized. These findings were similar to what has been reported in the caudate and putamen of human and non-human primates, as well as in rodents (Bennett and Bolam, 1993; Fortin and Parent, 1994; Parent et al., 1995, 1996; Cicchetti et al., 1998; Mura et al., 2000). In the human, an additional population of large-sized calretinin positive neurons has been observed in the dorsal striatum, even when these neurons represented only a small fraction of the calret-ir cell population (Parent et al., 1995; Cicchetti et al., 1998). In the squirrel monkey, this population of large-sized calretinin immunolabeled neurons has only been reported in the limbic striatum, outside of the caudate and putamen nuclei (Parent et al., 1996). Large-sized calret-ir neurons have been tentatively identified as large cholinergic striatal interneurons (Parent et al., 1995; Cicchetti et al., 1998) and may be restricted to the ventral striatum of the tree shrew, similar to what has been reported in the squirrel monkey (Parent et al., 1996). Even when calretinin expression in the tree shrew striatum presented some similarities with primates, there were also specific features, such as the presence of a clear difference in the distribution of calretinin labeling between the caudate and putamen, that have not been reported in primates (Fortin and Parent, 1994; Parent et al., 1995, 1996).

\section{STRIOSOME/MATRIX PATTERN IN THE TREE SHREW}

The striosome and matrix are two distinct cytoarchitectonic compartments consistently present in the striatum of many mammals including primates, felines, and rodents (see, e.g., Graybiel and Ragsdale, 1978; Graybiel et al., 1981; Gerfen, 1984; Donoghue and Herkenham, 1986; Desban et al., 1993; Parent et al., 1996; Holt et al., 1997; Prensa et al., 1999). The striosome/matrix organization was first visualized by Graybiel and Ragsdale (1978) using acetylcholinesterase histochemistry. In this work we showed that this organization is also present in the tree shrew striatum using calbindin immunolabeling, another well-established marker for these cytoarchitectonic compartments (see, e.g., Gerfen et al., 1985; Gerfen, 1992; Parent et al., 1996; Holt et al., 1997; Prensa et al., 1999). One prominent feature of the striosome/matrix organization in the tree shrew is the existence of two large striosomes in the dorsomedial caudate and dorsolateral putamen. This pattern may correspond with the increasing dorsoventral gradient that has been observed in human and non-human primates (Parent et al., 1996; Holt et al., 1997). On the contrary, in the rat, instead of a gradient, the dorsolateral caudate/putamen is free of calbindin neuropil, while calb-ir neurons are still present (see as a review Hontanilla et al., 1998). In agreement with our findings in the tree shrew, Prensa et al. (1999) reported that these large striosomes that occupy the dorsal half of the caudate nucleus were also present in humans. Also, in agreement with our results, Parent et al. (1996) reported that this striosome/matrix pattern became less apparent in more caudal sections. In fact, in our study we have observed that calb-ir decreased, but was still present in the striosomes to a certain degree. This observation is similar to what has been found in both squirrel monkey (Parent et al., 1996), and 
rat (Hontanilla et al., 1998). An extra layer of complexity in the striosome/matrix organization has been described using calbindin in the human striatum. In the posterior human striatum it has been reported that calbindin labeling produced a ring-like structure within the striosomes, and this could be interpreted as the presence of a "core" and a "shell" within the striosomes (Bernacer et al., 2008). We did not observe any ring-like structure in the tree shrew striatum, although there was a gradient of calbindin labeling toward the center of the striosomes (see Figures 5D,E). This gradient has also been observed in non-human primates such as the squirrel monkey (Levesque et al., 2004). Calbindin neuronal labeling in the tree shrew was similar to that found in non-human primates, with calbindin positive neurons displaying labeling in the cytoplasm and in the initial segments of one or more processes (Parent et al., 1996). Overall, the pattern of calbindin expression in the tree shrew striatum was remarkably similar to the expression of this marker in human and non-human primates. This supports that the striosome/matrix distribution observed in the tree shrew dorsal striatum is very similar to what has been reported in primates including humans.

Studies on tyrosine hydroxylase expression in the dorsal striatum have shown that this enzyme also reveals the striosome/matrix compartments in primates (Graybiel et al., 1987; Prensa et al., 1999; Stephenson et al., 2005; Bernacer et al., 2008), with the striosomes appearing as low $\mathrm{TH}$-ir areas. In the tree shrew striatum $\mathrm{TH}$-ir also revealed the striosome/matrix organization, and our detailed mapping showed that the distribution of these compartments matched almost exactly the organization recognized by calbindin labeling (see Figures 8-10). Similar results have been reported in the striatum of primates including humans (Graybiel et al., 1987; Prensa et al., 1999; Stephenson et al., 2005; Bernacer et al., 2008) but not in adult rodents (see, e.g., Hedou et al., 2000; Sutoo et al., 2002). On the other hand, it has been reported by Sutoo et al. (2002) that a gradient of TH staining was present in the striatum of the rodent, although this gradient did not correspond with a striosome/matrix organization.

Another salient feature of TH labeling in the tree shrew striatum was the presence of a discrete number of TH-ir cells that can be morphologically classified in three subtypes. The presence of discrete numbers of dopaminergic cells in primates including humans has been consistently reported (Dubach et al., 1987; Ikemoto et al., 1996; Betarbet et al., 1997; Cossette et al., 1999, 2004, 2005; Huot and Parent, 2007), while in rodents TH-ir cells are almost anecdotal in the normal striatum (Tashiro et al., 1989; Mura et al., 1995; Baker et al., 2003; Huot and Parent, 2007). In addition, as we observed in the tree shrew (present results), dopaminergic cells in the primate striatum presented a variety of subtypes readily distinguishable by their size and other morphological features (Dubach et al., 1987; Betarbet et al., 1997; Cossette et al., 2005; Huot and Parent, 2007), and it has been postulated that most of these TH-ir neurons are GABAergic interneurons (Betarbet et al., 1997). In summary, TH labeling in the tree shrew striatum strikingly resembled the pattern observed in primates and clearly diverged with the expression of $\mathrm{TH}$ in rodents.

Acetylcholinesterase was the first enzyme found to reveal the striosome/matrix organization in the striatum. Histochemistry and immunohistochemistry against this enzyme, or immunohistochemistry against choline acetyltransferase (the ratelimiting enzyme for the production of acetylcholine), have been used to reveal these cytoarchitectonic compartments in primates as well as in several non-primate species (see, e.g., Graybiel and Ragsdale, 1978; Natuk and Graybiel, 1985; Graybiel et al., 1986; Loopuijt et al., 1987; Rhodes et al., 1987; Hirsch et al., 1989; Holt et al., 1996, 1997; Prensa et al., 1999; Bernacer et al., 2008).

In this study we used two different antibodies against acetylcholinesterase. One of the antibodies was designed to specifically recognize human AchE (see Materials and Methods). The second antibody has been reported to recognize AchE in rodents, felines, and rabbits, but not in primates (Rackonczay and Brimijoin, 1986). In our work we found that the antibody that recognizes AchE only in non-primates did not produce any positive results in the tree shrew tissue, while producing positive results in rat striatal sections incubated together. On the contrary, the antibody specifically produced to recognize human AchE yielded positive results in the tree shrew. This finding may indicate that tree shrew acetylcholinesterase is closer to the primate forms of this enzyme than to the forms present in rodents and other non-primate species. Acetylcholinesterase immunolabeling in the tree shrew presented a similar pattern to what has been shown in primates including humans (see, e.g., Graybiel and Ragsdale, 1978; Natuk and Graybiel, 1985; Hirsch et al., 1989; Holt et al., 1996, 1997; Prensa et al., 1999; Bernacer et al., 2008). Our results also showed that the striosomes identified by AchE immunolabeling aligned in a great manner with the striosomal organization revealed by calbindin and TH (see Figures 8-10, present results). Comparison of the three striosome/matrix markers used in the present study clearly demonstrated that the tree shrew also possesses these cytoarchitectonic compartments. Our results also showed that calbindin and tyrosine hydroxylase were the most effective markers for the delineation of these compartments in the tree shrew, although acetylcholinesterase antibodies also revealed a very similar striosome/matrix organization.

\section{LABELING FOR GAD 65/67 AND MORPHOLOGY OF MEDIUM SPINY NEURONS}

The major cell type of the mammalian dorsal striatum is the medium spiny GABAergic neuron. This type of projection neurons constitute up to $95 \%$ of the total number of neurons in the striatum of felines and rodents (Kemp and Powell, 1971; Graveland and DiFiglia, 1985; see as a review Voogd et al., 1998) and approximately $75 \%$ in the primate striatum (Graveland and DiFiglia, 1985; see as reviews Nieuwenhuys et al., 2007; Tepper et al., 2010). GABA in the striatum is expressed in the medium spiny projection neurons as well as in local interneurons (Cowan et al., 1990; Kita et al., 1990; Kawaguchi, 1993; Kubota et al., 1993; Kubota and Kawaguchi, 2000; see as reviews, Nieuwenhuys et al., 2007; Tepper et al., 2010), producing a very rich and intricate network of GABAergic cell bodies and processes.

In the tree shrew dorsal striatum GAD 65/67 labeling consisted of densely packed and strongly labeled puncta. Even when they were rarely observed, GAD 65/67 positive neurons were also present and displayed cytoplasmic granular staining. Cellular immunolabeling is difficult to detect with anti-GAD 65/67 antibodies in the striatum under normal conditions, due to the 
rapid transportation of GAD from the soma to processes and terminal buttons (Ribak et al., 1978, 1979; Kita and Kitai, 1988). The difficulty associated with the observation of GAD neuronal staining has also been reported in primates and rodents (see, e.g., Levesque et al., 2004; Stephenson et al., 2005). GAD soma staining can only be clearly observed in a high number of neurons after using colchicine, a chemical treatment that prevents the rapid transport of GAD from the soma (Ribak et al., 1978, 1979; Kita and Kitai, 1988; Vuillet et al., 1990; Kubota et al., 1993). GAD $65 / 67$ labeling in the dorsal striatum of the tree shrew appeared as strong puncta immunolabeling that was homogeneously distributed and did not present a patch-like pattern or clear gradient. Levesque et al. (2004) reported that the expression of GAD resembled a patch-like pattern in primates, but not in rodents. However, studies using quantitative immunohistochemistry for GAD in the human striatum did not replicate these findings of a patch-like distribution (Sutoo et al., 2000). In this quantitative study by Sutoo et al. (2000), the only reported change in the intensity of GAD involved the presence of a dorsoventral decreasing gradient in the caudate, and a slight dorsoventral increasing gradient in the putamen. In support of this, Stephenson et al. (2005) neither reported nor showed any evidence of a patch-like distribution for GAD 65 or GAD 67 in the striatum of untreated monkeys. In view of these findings, a patch-like pattern of GAD immunolabeling in primates as a group appears to be rather controversial, with quantitative work in the human supporting a non-patch-like distribution of GAD staining (Sutoo et al., 2000). Although a qualitative observation of GAD labeling in the tree shrew striatum did not reveal any gradient of staining in the caudate or putamen, we can not discard the possibility that a quantitative analysis may reveal some slight differences between dorsal and ventral regions of these nuclei.

To further characterize the medium spiny GABAergic neurons in the tree shrew we used Golgi impregnation techniques. Our results showed that medium spiny cells in the tree shrew presented a round to polygonal soma and 5-6 primary dendrites that

\section{REFERENCES}

Adkins, R. M., and Honeycutt, R. L. (1991). Molecular phylogeny of the superorder Archonta. Proc. Natl. Acad. Sci. U.S.A. 88, 10317-10321.

Amsterdam, J. D., and Newberg, A. B. (2007). A preliminary study of dopamine transporter binding in bipolar and unipolar depressed patients and healthy controls. $\mathrm{Neu}$ ropsychobiology 55, 167-170.

Baker, H., Kobayashi, K., Okano, H., and Saino-Saito, S. (2003). Cortical and striatal expression of tyrosine hydroxylase mRNA in neonatal and adult mice. Cell. Mol. Neurobiol. 23, 507-518.

Bartolomucci, A., de Biurrun, G., and Fuchs, E. (2001). How tree shrews (Tupaia belangeri) perform in a searching task: evidence for strategy use. J. Comp. Psychol. 115, 344-350.

Bender, R. A., Lauterborn, J. C., Gall, C. M., Cariaga, W., and Baram, T. Z.

emanated from the cell body. Similar cell shapes and numbers of primary dendrites have been reported in medium spiny neurons of primates, felines, and rodents (Kemp and Powell, 1971; Mensah and Deadwyler, 1974; DiFiglia et al., 1976; Braak and Braak, 1982; Graveland et al., 1985; Yelnik et al., 1991). In our study we also showed that the average cross-section of the soma was $273.26 \mu \mathrm{m}^{2}$, and that the mean distance from the soma to secondary dendrites was $15.86 \mu \mathrm{m}$. These measurements were in the same range that has been observed in primates (DiFiglia et al., 1976; Braak and Braak, 1982; Graveland et al., 1985; Yelnik et al., 1991), while in rodents and in the cat, the soma size for this type of cells varies in a larger manner (10-20 $\mu \mathrm{m}$ of diameter in the rat, and $12-18 \mu \mathrm{m}$ in the cat, which assuming an spheroid shape for the soma is equivalent to cross-sections of $78-314$ and $113-254 \mu \mathrm{m}^{2}$, in the rat and cat respectively; Kemp and Powell, 1971; Mensah and Deadwyler, 1974). In addition, as we report here for the tree shrew, in some rare cases dendritic spines were observed in the soma of medium spiny neurons in primates (DiFiglia et al., 1976; Graveland et al., 1985).

Overall, the neurochemical characterization of the tree shrew dorsal striatum revealed striking similarities in the distribution of most of the markers studied when compared to primates, while presenting more marked divergences compared to the rodent striatum. Our results suggest that the tree shrew striatum could represent a good animal model for studies of the basal ganglia aimed at the analysis of the expression of neurotransmitters or other molecules in normal or diseased brain.

\section{ACKNOWLEDGMENTS}

The authors wish to thank Dr. Thomas Norton and Dr. John Siegwart, Jr. (Department of Vision Sciences, University of Alabama at Birmingham) for providing us with the tree shrew brains used in this study. We also thank Ms. Rosie Ricks for her helpful editing of the final manuscript. Supported by, NIH-NIMH RO1MH060744 grant to Rosalinda C. Roberts.

P-immunoreactive terminals in the rat neostriatum with particular reference to identified spiny striatonigral neurons. Exp. Brain Res. 70, 361-377

Bolam, J. P., Izzo, P. N., and Graybiel, A. M. (1988). Cellular substrate of the histochemically defined striosome/matrix system of the caudate nucleus: a combined golgi and immunocytochemical study in the cat and ferret. Neuroscience 24, 853-875.

Bolam, J. P., and Smith, Y. (1990). The GABA and substance $P$ input to dopaminergic neurons in the substantia nigra of the rat. Exp. Brain Res. 529, 57-78.

Braak, H., and Braak, E. (1982). Neuronal types in the striatum of man. Cell Tissue Res. 227, 319-342.

Buchsbaum, M. S., Potkin, S. G., Siegel, B. V. Jr., Lohr, J., Katz, M., Gottschalk, L. A., Gulasekaram, B., Marshall, J.
F., Lottenberg, S., Teng, C. Y., Abel, L., Plon, L., and Bunney, W. E. Jr. (1992). Striatal metabolic rate and clinical response to neuroleptics in schizophrenia. Arch. Gen. Psychiatry 49, 966-974.

Butters, M. A., Aizenstein, H. J., Hayashi, K. M., Meltzer, C. C., Seaman, J., Reynolds, C. F., Toga, A. W., Thompson, P. M., Becker, J. T., and IMAGe Research Group. (2009). Three-dimensional surface mapping of the caudate nucleus in late-life depression. Am. J. Geriatr. Psychiatry 17, 4-12.

Cao, J., Yang, E. B., Su, J. J., Li, Y., and Chow, P. (2003). The tree shews: adjuncts and alternatives to primates as models for biomedical research. $J$. Med. Primatol. 32, 123-130.

Celio, M. R. (1986). Parvalbumin in most gamma-aminobutyric acidcontaining neurons of the rat cerebral cortex. Science 231, 995-997. 
Chomsung, R. D., Wei, H., Day-Brown, J. D., Petry, H. M., and Bickford, M. E. (2010). Synaptic organization of connections between the temporal cortex and pulvinar nucleus of the tree shrew. Cereb. Cortex 20, 997-1011.

Cicchetti, F., Beach, T. G., and Parent, A. (1998). Chemical phenotype of calretinin interneurons in the human striatum. Synapse 30, 284-297.

Cohen, J. (2007a). Biomedical research. The endangered lab chimp. Science $315,450-452$.

Cohen, J. (2007b). Animal studies. NIH to end chimp breeding for research. Science 316, 1265.

Cossette, M., Lecomte, F., and Parent, A. (2005). Morphology and distribution of dopaminergic neurons intrinsic to the human striatum. $J$. Chem. Neuroanat. 29, 1-11.

Cossette, M., Levesque, M., and Parent, A. (1999). Extrastriatal dopaminergic innervation of human basal ganglia. Neurosci. Res. 34, 51-54.

Cossette, M., Parent, A., and Levesque, D. (2004). Tyrosine hydroxylasepositive neurons intrinsic to the human striatum express the transcription factor Nurrl. Eur. J. Neurosci. 20, 2089-2095.

Cote, P. Y., Sadikot, A. F., and Parent, A. (1991). Complementary distribution of calbindin D-28k and parvalbumin in the basal forebrain and midbrain of the squirrel monkey. Eur. J. Neurosci. 3, 1316-1329.

Cousins, D. A., Butts, K., and You, A. H. (2009). The role of dopamine in bipolar disorder. Bipolar Disord 11, 787-806.

Cowan, R. L., Wilson, C. J., Emson, P. C., and Heizmann, C. W. (1990). Parvalbumin-containing GABAergic interneurons in the rat neostriatum. J. Comp. Neurol. 302, 197-205.

Czeh, B., Michaelis, T., Watanabe, T. Frahm, J., de Biurrun, G., van Kampen, M., Bartolomucci, A., and Fuchs, E. (2001). Stress-induced changes in cerebral metabolites, hippocampal volume, and cell proliferation are prevented by antidepressant treatment with tianeptine. Proc. Natl. Acad. Sci. U.S.A. 98, 12796-12801.

Day-Brown, J. D., Wei, H., Chomsung, R. D., Petry, H. M., and Bickford, M. E. (2010). Pulvinar projections to the striatum and amygdala in the tree shrew. Front. Neuroanat. 4:143. doi: 10.3389/fnana.2010.00143.

De las Heras, S., Hontanilla, B., Mengual, E., and Gimenez-Amaya, J. M. (1994). Immunohistochemical distribution of calbindin D-28k and parvalbumin in the head of the caudate nucleus and substantia nigra of the cat. J. Morphol. 221, 291-307.

DeLong, M., Alexander, G. E., Georgopoulos, A. P., Crutcher, M. D., Mitchell, S. J., and Richardson, R. T. (1984). Role of basal ganglia in limb movements. Hum. Neurobiol. 2, 235-244.

DeLong, M. R., and Georgopoulos, A. P. (1981). Motor functions of the basal ganglia. Handb. Physiol. 3, 1017-1061.

Desban, M., Gauchy, C., Glowinski, J., and Kemel, M. L. (1995). Heterogeneous topographical distribution of the striatonigral and striatopallidal neurons in the matrix compartment of the cat caudate nucleus. J. Comp. Neurol. 352, 117-133.

Desban, M., Kemel, M. L., Glowinski, J., and Gauchy, C. (1993). Spatial organization of patch and matrix compartments in the rat striatum. Neuroscience 57, 661-671.

Dickson, D. W., Crystal, H. A., Mattiace, L. A., Masur, D. M., Blau, A. D., Davies, P., Yen, S. H., and Aronson, M. K. (1992). Identification of normal and pathological aging in prospectively studied nondemented elderly humans. Neurobiol. Aging 13, 179-189.

DiFiglia, M., and Aronin, N. (1982). Ultrastructural features of immunoreactive somatostatin neurons in the rat caudate nucleus. J. Neurosci. 2, 1267-1274.

DiFiglia, M., Pasik, P., and Pasik, T. (1976). A golgi study of neuronal types in the neostriatum of monkeys. Brain Res. 114, 245-256.

Divac, I., and Passingham, R. E. (1980). Connections of the mediodorsal nucleus of the thalamus in the tree shrew. II. Efferent connections. Neurosci. Lett. 19, 21-26.

Donoghue, J. P., and Herkenham, M. (1986). Neostriatal projections from individual cortical fields conform to histochemically distinct striatal compartments in the rat. Brain Res. $365,397-403$.

Dubach, M., Schmidt, R., Kunkel, D., Bowden, D. M., Martin, R., and German, D. C. (1987). Primate neostriatal neurons containing tyrosine hydroxylase: immunohistochemical evidence. Neurosci. Lett. 75, 205-210.

Dube, L., Smith, A. D., and Bolam, J. P. (1988). Identification of synaptic terminals of thalamic or cortical origin in contact with distinct medium-size spine neurons in the rat neostriatum. J. Comp. Neurol. $267,455-471$.

Elston, G. N., Elston, A., Casagrande, V., and Kaas, J. H. (2005). Areal special- ization of pyramidal cell structure in the visual cortex of the tree shrew: a new twist revealed in the evolution of cortical circuitry. Exp. Brain Res. $163,13-20$.

Figueredo-Cardenas, G., Medina, L., and Reiner, A. (1996). Calretinin is largely localized to a unique population of striatal interneurons in rats. Brain Res. 709, 145-150.

Fitzpatrick, D. (1996). The functional organization of local circuits in visual cortex: insights from the study of tree shrew striate cortex. Cereb. Cortex 6, 329-341.

Fonnum, F., Storm-Mathisen, J., and Divac, I. (1981). Biochemical evidence for glutamate as neurotransmitter in corticostriatal and corticothalamic fibres in rat brain. $\mathrm{Neu}$ roscience 6, 863-873.

Fortin, M., and Parent, A. (1994). Patches in the striatum of squirrel monkeys are enriched with calretinin fibers but devoid of calretinin cell bodies. Neurosci. Lett. 182, 51-54.

Fuchs, E. (2005). Social stress in tree shrews as an animal model of depression: an example of behavioral model of a CNS disorder. CNS Spectr. 10, 182-190.

Fuchs, E., and Flugge, G. (2002). Social stress in tree shrews: effects on physiology, brain function, and behavior of subordinate individuals. Pharmacol. Biochem. Behav. 73 247-258.

Fuchs, E., Uno, H., and Flugge, G. (1995). Chronic psychosocial stress induces morphological alterations in hippocampal pyramidal neurons of the tree shrew. Brain Res. 673 , 275-282.

Fudge, J. L., and Haber, S. N. (2002). Defining the caudal ventral striatum in primates: cellular and histochemical features. J. Neurosci. 22, 10078-10082.

Fujiyama, F., Unzai, T., Nakamura, K., Nomura, S., and Kaneko, T. (2006). Difference in organization of corticostriatal and thalamostriatal synapses between patch and matrix compartments of rat neostriatum. Eur. J. Neurosci. 24, 2813-2824.

Gerfen, C. R. (1984). The neostriatal mosaic: compartmentalization of corticostriatal input and striatonigral output systems. Nature 311, 461-464.

Gerfen, C. R. (1989). The neostriatal mosaic: striatal patch-matrix organization is related to cortical lamination. Science 246, 385-388.

Gerfen, C. R. (1992). The neostriatal mosaic: multiple levels of compartmental organization in the basal ganglia. Annu. Rev. Neurosci. 15 285-320.

Gerfen, C. R., Baimbridge, K. G., and Miller, J. J. (1985). The neostriatal mosaic: compartmental distribution of calcium-binding protein and parvalbumin in the basal ganglia of the rat and monkey. Proc. Natl. Acad. Sci. U.S.A. 82, 8780-8784.

Gimenez-Amaya, J. M., and Graybiel, A. M. (1990). Compartmental origins of the striatopallidal projection in the primate. Neuroscience 34 , 111-126.

Gould, E., McEwen, B. S., Tanapat, P., Galea, L. A., and Fuchs, E. (1997). Neurogenesis in the dentate gyrus of the adult tree shrew is regulated by psychosocial stress and NMDA receptor activation. J. Neurosci. 17, 2492-2498.

Graveland, G. A., and DiFiglia, M. (1985). The frequency and distribution of medium-sized neurons with indented nuclei in the primate and rodent neostriatum. Brain Res. 327 307-311.

Graveland, G. A., Williams, R. S., and DiFiglia, M. (1985). A golgi study of the human neostriatum: neurons and afferent fibers. J. Comp. Neurol. 234, 317-333.

Graybiel, A. M. (1990). Neurotransmitters and neuromodulators in the basal ganglia. Trends Neurosci. 13, 244-254.

Graybiel, A. M., Baughman, R. W., and Eckenstein, F. (1986). Cholinergic neuropil of the striatum observes striosomal boundaries. Nature 323, 625.627.

Graybiel, A. M., Flaherty, A. W., and Gimenez-Amaya, J. M. (1991). "Striosomes and matrisomes," in The Basal Ganglia, Vol. 3, eds G. Bernardi, M. B. Carpenter, G. di Chiara, M. Morelli, and P. Stanzione (New York: Plenum), 3-12.

Graybiel, A. M., Hirsch, E. C., and Agid, Y. A. (1987). Differences in tyrosine hydroxylase-like immunoreactivity characterize the mesostriatal innervation of striosomes and extrastriosomal matrix at maturity. Proc. Natl. Acad. Sci. U.S.A. 84, 303-307.

Graybiel, A. M., and Ragsdale, C. W. (1978). Histochemically distinct compartments in the striatum of human, monkey, and cat demonstrated by acetylcholinesterase staining. Proc. Natl. Acad. Sci. U.S.A. 75 , 5723-5726.

Graybiel, A. M., and Ragsdale, C. W. (1983). "Biochemical anatomy of the striatum," in Chemical Neuroanatomy, ed. P. C. Emson (New York: Raven Press), 427-504. 
Grabyiel, A. M., Ragsdale, C. W., Yoneoka, E. S., and Elde, R. H. (1981). An immunohistochemical study of enkephalins and other neuropeptide in the striatum of the cat with evidence that the opiate peptides are arranged to form mosaic patterns in register with the striosomal compartments visible by acetylcholinesterase staining. Neuroscience 6, 377-397.

Haber, S., and Elde, R. (1982). The distribution of enkephalin immunoreactive fibers and terminals in the monkey central nervous system: an immunohistochemical study. $\mathrm{Neu}$ roscience 7, 1049-1095.

Haber, S. N., and McFarland, N. R. (1999). The concept of the ventral striatum in nonhuman primates. Ann. N. Y. Acad. Sci. 877, 33-48.

Hedou, G., Chasserot-Golaz, S., Kemmel, V., Gobaille, S., Roussel, G., Artault, J. C., Andriamampandry, C., Aunis, D., and Maitre, M. (2000). Immunohistochemical studies of the localization of neurons containing the enzyme that synthesizes dopamine, GABA, or gammahydroxybutyrate in the rat substantia nigra and striatum. J. Comp. Neurol. 426, 549-560.

Heimer, L., Switzer, R. D., and Van Hoesen, G. W. (1982). Ventral striatum and ventral pallidum. Components of the motor system? Trends Neurosci. 5, 83-87

Heimer, L., and Wilson, R. D. (1975). "The subcortical projections of the allocortex: similarities in the neural associations of the hippocampus, the piriform cortex, and the neocortex," in Golgi Centennial Symposium: Perspectives in Neurobiology, ed. M. Santini (New York: Raven Press), 177-193.

Hietala, J., Syvalahti, E., Vuorio, K., Rakkolainen, V., Bergman, J., Haaparanta, M., Solin, O., Kuoppamaki, M., Kirvela, O., Ruotsalainen, U., and Salokangas, R. K. R. (1995). Presynaptic dopamine function in striatum of neuroleptic-naïve schizophrenic patients. Lancet 346, 1130-1131.

Hiroi, N. (1995). Compartmental organization of calretinin in the rat striatum. Neurosci. Lett. 197, 223-226.

Hirsch, E. C., Graybiel, A. M., Hersh, L. B., Duyckaerts, C., and Agid, Y. (1989). Striosomes and extrastriosomal matrix contain different amounts of immunoreactive choline acetyltransferase in the human striatum. Neurosci. Lett. 96, 145-150.

Holt, D. J., Graybiel, A. M., and Saper, C. B. (1997). Neurochemical architecture of the human striatum. J. Comp. Neurol. 384, $1-25$.

Holt, D. J., Hersh, L. B., and Saper, C. B. (1996). Cholinergic innervation in the human striatum: a threecompartment model. Neuroscience 74, 67-87.

Hontanilla, B., Parent, A., de las Heras, S., and Giménez-Amaya, J. M. (1998). Distribution of calbindin D$28 \mathrm{k}$ and parvalbumin neurons and fibers in the rat basal ganglia. Brain Res. Bull. 47, 107-116.

Howes, O. D., Montgomery, A. J., Asselin, M. C., Murray, R. M., Valli, I., Tabraham, P., Bramon-Bosch, E., Valmaggia, L., Johns, L., Broome, M., McGuire, P. K., and Grasby, P. M. (2009). Elevated striatal dopamine function linked to prodromal signs of schizophrenia. Arch. Gen. Psychiatry 66, 13-20.

Huot, P., and Parent, A. (2007). Dopaminergic neurons intrinsic to the striatum. J. Neurochem. 101, 1441-1447.

Ikemoto, K., Satoh, K., Kitahama, K., and Maeda, T. (1996). Demonstration of a new dopamine-containing cell group in the primate rostral telencephalon. Neurosci. Lett. 220, 69-71.

Isovich, E., Mijnster, M. J., Flugge, G., and Fuchs, E. (2000). Chronic psychosocial stress reduces the density of dopamine transporters. Eur. J. Neurosci. 12, 1071-1078.

Janecka, J. E., Miller, E., Pringle, T. H., Wiens, F., Zitzmann, A., Helgen, K. M., Springer, M. S., and Murphy, W. J. (2007). Molecular and genomic data identify the closest living relative of primates. Science 318, 792-794.

Joel, D., and Weiner, I. (2000). The connections of the dopaminergic system with the striatum in rats and primates: an analysis with respect to the functional and compartmental organization of the striatum. $\mathrm{Neu}$ roscience $96,451-474$.

Karnovsky, M. J., and Roots, L. (1964). A direct coloring thiocholine method for cholinesterases. J. Histochem. Cytochem. 12, 219-221.

Kataoka, Y., Kalanithi, P. S. A., Grantz, H., Schwartz, M. L., Saper, C., Leckman, J. F., and Vaccarina, F. M. (2010). Decreased number of parvalbumin and cholinergic interneurons in the striatum of individuals with Tourette syndrome. J. Comp. Neurol. 518, 277-291.

Kawaguchi, Y. (1993). Physiological, morphological, and histochemical characterization of three classes of interneurons in rat neostriatum. $J$. Neurosci. 13, 4908-4923.

Kawaguchi, Y., and Hirano, T. (2002). Signaling cascade regulating longterm potentiation of GABAA receptor responsiveness in cerebellar Purkinje neurons. J. Neurosci. 22, 3969-3976.

Kawaguchi, Y., Wilson, C. J., Augood, S. J., and Emson, P. C. (1995). Striatal interneurones: chemical, physiological and morphological characterization. Trends Neurosci. 18, 527-535.

Kemp, J. M., and Powell, T. P. (1971). The structure of the caudate nucleus of the cat: light and electron microscopy. Philos. Trans. R. Soc. Lond. B Biol. Sci. 262, 383-401.

Keuker, J. I., Rochford, C. D., Witter, M. P., and Fuchs, E. (2003). A cytoarchitectonic study of the hippocampal formation of the tree shrew (Tupaia belangeri). J. Chem. Neuroanat. 26, $1-15$.

Khundakar, A., Morris, C., Oakley, A., and Thomas, A. J. (2011). Morphometric analysis of neuronal and glial cell pathology in the caudate nucleus in late-life depression. Am. J. Geriatr. Psychiatry 19, 132-141.

Kita, H., and Kitai, S. T. (1988). Glutamate decarboxylase immunoreactive neurons in rat neostriatum: their morphological types and populations. Brain Res. 447, 346-352.

Kita, H., Kosaka, T., and Heizmann, C. W. (1990). Parvalbuminimmunoreactive neurons in the rat neostriatum: a light and electron microscopic study. Brain Res. 536, $1-15$.

Kubota, Y., Inagaki, S., and Kito, S. (1986). Innervation of substance $P$ neurons by catecholaminergic terminals in the neostriatum. Brain Res. 375, 163-167.

Kubota, Y., and Kawaguchi, Y. (1993). Spatial distributions of chemically identified intrinsic neurons in relations to patch and matrix compartments of rat neostriatum. J. Comp. Neurol. 332, 499-513.

Kubota, Y., and Kawaguchi, Y. (2000). Dependence of GABAergic synaptic areas on interneuron type and target size. J. Neurosci. 20, 375-386.

Kubota, Y., Mikawa, S., and Kawaguchi, Y. (1993). Neostriatal GABAergic interneurones contain NOS, calretinin or parvalbumin. Neuroreport 5 205-208.

Lavoie, B., and Parent, A. (1990). Immunohistochemical study of the serotonergic innervation of the basal ganglia in the squirrel monkey. $J$. Comp. Neurol. 299, 1-16.
Levesque, M., Wallman, M. J., and Parent, A. (2004). Striosomes are enriched in glutamic acid decarboxylase in primate. Neurosci. Res. 50, 29-35.

Lin, C. S., May, P. J., and Hall, W. C. (1984). Nonintralaminar thalamostriatal projections in the grey squirrel (Sciurus carolinensis) and tree shrew (Tupaia glis). J. Comp. Neurol. 230, 33-46.

Liu, F. C., and Graybiel, A. M. (1992). Heterogeneous development of calbindin-D28k expression in the striatal matrix. J. Comp. Neurol. 320, 304-322.

Liu, F. G. R., Miyamoto, M. M., Freire, N. P., Ong, P. Q., Tennant, M. R., Young, T. S., and Gugel, K. F. (2001). Molecular and morphological supertrees for Eutherian (placental) mammals Science 291, 1786-1789.

Loopuijt, L. D., Sebens, J. B., and Korf, J. (1987). A mosaic-like distribution of dopamine receptors in rat neostriatum and its relationship to striosomes. Brain Res. 405, 405-408.

Luckett, W. P. (1980). Comparative Biology and Evolutionary Relationships of Tree Shrews. New York: Plenum Press.

Lyon, D. C., Jain, N., and Kaas, J. H. (2003). The visual pulvinar in tree shrews I. Multiple subdivision revealed through acetylcholinesterase and Cat-301 chemoarchitecture. J. Comp. Neurol. 467, 593-606.

Mai, J. K., Assheuer, J., and Paxinos, G. (2004). Atlas of the Human Brain, 2nd Edn. San Diego: Elsevier Academic Press.

Marsden, C. D. (1986). Movement disorders and the basal ganglia. Trends Neurosci. 32, 512-515.

Matsuo, K., Rosenberg, D. R., Easter, P. C., MacMaster, F. P., Chen, H. H., Nicoletti, M., Caetano, S. C., Hatch, J. P., and Soares, J. C. (2008). Striatal volume abnormalities in treatmentnaïve patients diagnosed with pediatric major depressive disorder. J. Child Adolesc. Psychopharmacol. 18, 121-131.

McCoy, P., Norton, T. T., and McMahon, L. L. (2008). Layer 2/3 synapses in monocular and binocular regions of tree shrew visual cortex express mAChR-dependent long-term depression and long-term potentiation. J. Neurophysiol. 100, 336-345.

Meier, J., and Grantyn, R. (2004) A gephyrin-related mechanism restraining glycine receptor anchoring at GABAergic synapses. J. Neurosci. 24, 1398-1405. 
Melendez-Ferro, M., Perez-Costas, E., and Roberts, R. C. (2009). A new use for long-term frozen brain tissue: golgi impregnation. J. Neurosci. Methods 176, 72-77.

Mensah, P., and Deadwyler, S. (1974). The caudate nucleus of the rat: cell types and the demonstration of a commissural system. J. Anat. 117, 281-293.

Middleton, F. A., and Strick, P. L. (1994). Anatomical evidence for cerebellar and basal ganglia involvement in higher cognitive function. Science $226,452-461$.

Mijnster, M. J., Isovich, E., Flugge, G., and Fuchs, E. (1999). Localization of dopamine receptors in the tree shrew brain using [3H]-SCH23390 and [125I]-epidepride. Brain Res. 841, 101-113.

Morel, A., Loup, F., Magnin, M., and Jeanmonod, D. (2002). Neurochemical organization of the human basal ganglia: anatomofunctional territories defined by the distributions of calcium-binding proteins and SMI32. J. Comp. Neurol. 443, 86-103.

Mura, A., Feldon, J., and Mintaz, M. (2000). The expression of the calcium binding protein calretinin in the rat striatum: effects of dopamine depletion and L-DOPA treatment. Exp. Neurol. 164, 322-332.

Mura, A., Jackson, D., Manley, M. S., Young, S. J., and Groves, P. M. (1995). Aromatic L-amino acid decarboxylase immunoreactive cells in the rat striatum: a possible site for the conversion of exogenous L-DOPA to dopamine. Brain Res. 704, 51-60.

Murphy, W. J., Eizirik, E., O’Brien, S. J., Madsen, O., Scally, M., Douady, C. J., Teeling, E., Ryder, O. A., Stanhope, M. J., de Jong, W. W., and Springer, M. S. (2001). Resolution of the early placental mammal radiation using Bayesian phylogenetics. Science 294, 2348-2351.

Nafia, I., Re, D. B., Masmejean, F., Melon, C., Kachidian, P., Goff, L. K., Nieoullon, A., and Had-Aissouni, L. (2008). Preferential vulnerability of mesencephalic dopamine neurons to glutamate transporter dysfunction. J. Neurochem. 105, 484-496.

Natuk, M. A., and Graybiel, A. M. (1985). Patterns of muscarinic cholinergic binding in the striatum and their relation to dopamine islands and striosomes. J. Comp. Neurol. 237, 176-194.

Nieuwenhuys, R., Voogd, J., and van Huijzen, C. (2007). The Human Central Nervous System, 4th Edn. Berlin: Springer-Verlag.
Oliver, D. L. (1982). A golgi study of the medial geniculate body in the tree shrew (Tupaia glis). J. Comp. Neurol. 209, 1-16.

Parent, A., Cicchetti, F., and Beach, T. G. (1995). Calretinin-immunoreactive neurons in the human striatum. Brain Res. 674, 347-351.

Parent, A., Fortin, M., Cote, P. Y., and Cicchetti, F. (1996). Calciumbinding proteins in primate basal ganglia. Neurosci. Res. 25, 309-334.

Parent, A., and Hazrati, L. N. (1995) Functional anatomy of the basal ganglia. I. The cortico-basal gangliathalamo-cortical loop. Brain Res. Rev. 20, 91-127.

Paxinos, G., and Watson, C. (1998). The Rat Brain in Stereotaxic Coordinates, 4th Edn. New York: Academic Press.

Perez-Costas, E., Melendez-Ferro, M., and Roberts, R. C. (2010). Basal ganglia pathology in schizophrenia: dopamine connections and anomalies. J. Neurochem. 113 , 287-302.

Phelps, P. E., Houser, C. R., and Vaughn, J. E. (1985). Immunocytochemical localization of choline acetyltransferase within the rat neostriatum: a correlated light and electron microscopic study of cholinergic neurons and synapses. J. Comp. Neurol. 238 , 286-307.

Poveda, A., and Kretz, R. (2009). C-Fos expression in the visual system of the tree shrew (Tupaia belangeri). J. Chem. Neuroanat. 37, 214-228.

Prensa, L., Gimenez-Amaya, J. M. and Parent, A. (1998). Morphological features of neurons containing calcium-binding proteins in the human striatum. J. Comp. Neurol. 390, 552-563.

Prensa, L., Gimenez-Amaya, J. M., and Parent, A. (1999). Chemical heterogeneity of the striosomal compartment in the human striatum. $J$. Comp. Neurol. 413, 603-618.

Puelles, L., Milan, F. J., and Martinez de la Torre, M. (1996). A segmental map of architectonic subdivisions in the diencephalon of the frog Rana perezi: acetylcholinesterasehistochemical observations. Brain Behav. Evol. 47, 279-310.

Rackonczay, Z., and Brimijoin, S. (1986). Monoclonal antibodies to rat brain acetylcholinesterase: comparative affinity for soluble and membrane-associated enzyme and for enzyme from different vertebrate species. J. Neurochem. 46 , 280-287.

Ragsdale, C. W. Jr., and Graybiel, A. M. (1988). Fibers from the basolateral nucleus of the amygdala selectively innervate striosomes in the caudate nucleus of the cat. J. Comp. Neurol. 269, 506-522.

Rajakumar, N., Elisevich, K., and Flumerfelt, B. A. (1993). Compartmental origin of the striatoentopeduncular projection in the rat. J. Comp. Neurol. 331, 286-296.

Reiner, A., Medina, L., and Haber, S. N. (1999). The distribution of dynorphinergic terminals in striatal target regions in comparison to the distribution of substance P-containing and enkephalinergic terminals in monkeys and humans. Neuroscience 88 775-793.

Remple, M. S., Reed, J. L., Stepniewska, I., Lyon, D. C., and Kaas, J. H. (2007). The organization of frontoparietal cortex in the tree shrew (Tupaia belangeri): II. Connectional evidence for a frontal-posterior parietal network. J. Comp. Neurol. 501, 121-149.

Rhodes, K. J., Joyce, J. N., Sapp, D. W., and Marshall, J. F. (1987). $[3 \mathrm{H}]$ hemicholinium-3 binding in rabbit striatum: correspondence with patchy acetylcholinesterase staining and a method for quantifying striatal compartments. Brain Res. 412, 400-404.

Ribak, C. E., Vaughn, J. E., and Roberts, E. (1979). The GABA neurons and their axon terminals in the rat corpus striatum as demonstrated by GAD immunocytochemistry. J. Comp. Neurol. 187, 261-283.

Ribak, C. E., Vaughn, J. E., and Saito, K. (1978). Immunocytochemical localization of glutamic acid decarboxylase in neuronal somata following colchicine inhibition of axonal transport. Brain Res. 140, 315-332.

Roberts, R. C., Conley, R., Kung, L., Peretti, F. J., and Chute, D. J. (1996). Reduced striatal spine size in schizophrenia: a postmortem ultrastructural study. Neuroreport 7 , 1214-1218.

Roberts, R. C., Roche, J. K., and Conley, R. (2005). Synaptic differences in postmortem striatum of subjects with schizophrenia: a stereological ultrastructural analysis. Synapse 56 , 185-197.

Rostkowski, A. B., Teppen, T. L., Peterson, D. A., and Urban, J. H. (2009). Cell-specific expression of neuropeptide Y Y1 receptor immunoreactivity in the rat basolateral amygdala. J. Comp. Neurol. 517, 166-176.

Royce, G. J., and Laine, E. J. (1984) Efferent connections of the caudate nucleus, including cortical projections of the striatum and other basal ganglia: an autoradiographic and horseradish peroxidase investigation in the cat. J. Comp. Neurol. 226, 28-49.

Rushlow, W., Flumerfelt, B. A., and Naus, C. C. (1995). Colocalization of somatostatin, neuropeptide $\mathrm{Y}$, and NADPH-diaphorase in the caudateputamen of the rat. J. Comp. Neurol. 351, 499-508.

Selemon, L. D., and Goldman-Rakic, P. S. (1990). Topographic intermingling of striatonigral and striatopallidal neurons in the rhesus monkey. J. Comp. Neurol. 297, 359-376.

Simpson, E. H., Kellendonk, C., and Kandel, E. (2010). A possible role for the striatum in the pathogenesis of the cognitive symptoms of schizophrenia. Neuron 65, 585-596.

Smith, Y., Bevan, M. D., Shink, E., and Bolam, J. P. (1998). Microcircuitry of the direct and indirect pathways of the basal ganglia. Neuroscience 86 , 353-387.

Stephenson, D. T., Li, Q., Simmons, C., Connell, M. A., Meglasson, M. D., Merchant, K., and Emborg, M. E. (2005). Expression of GAD65 and GAD67 immunoreactivity in MPTP-treated monkeys with or without L-Dopa administration. Neurobiol. Dis. 20, 347-359.

Sutoo, D., Akiyama, K., and Yabe, K. (2000). Quantitative maps of GABAergic and glutamatergic neuronal systems in the human brain. Hum. Brain Mapp. 11, 93-103.

Sutoo, D., Akiyama, K., and Yabe, K. (2002). Comparison analysis of distributions of tyrosine hydroxylase, calmodulin and calcium/calmodulin-dependent protein kinase II in a triple stained slice of rat brain. Brain Res. 933, 1-11.

Tashiro, Y., Sugimoto, T., Hattori, T., Uemura, Y., Nagatsu, I., Kikuchi, H., and Mizuno, N. (1989). Tyrosine hydroxylase-like immunoreactive neurons in the striatum of the rat. Neurosci. Lett. 97, 6-10.

Tepper, J. M., Tecuapetla, F., Koos, T., and Ibanez-Sandoval, O. (2010). Heterogeneity and diversity of striatal GABAergic interneurons. Front. Neuroanat. 4:150. doi 10.3389/fnana.2010.00150.

Vincent, S. R., Johansson, O., Hokfelt, T., Skirboll, L., Elde, R. P., Terenius, L., Kimmel, J., and Goldstein, M. (1983). NADPH-diaphorase: a selective histochemical marker for striatal neurons containing both somatostatin and avian pancreatic polypeptide (APP)-like immunoreactivities. J. Comp. Neurol. 217, 252-263. 
Vogel, G. (2010). Animal research. Long-fought compromise reached on European animal rules. Science 329, 1588-1589.

Voogd, J., Nieuwenhuys, R., van Dongen, P. A. M., and Ten Donkelaar, H. J. (1998). "Mammals," in The Central Nervous System of Vertebrates, eds R. Nieuwenhuys, H. J. Ten Donkelaar, and C. Nicholson (Berlin: Springer-Verlag), 1637-2097.

Vuillet, J., Kerkerian-Le Goff, L., Kachidian, P., Dusticier, G., Bosler, O., and Nieoullon, A. (1990). Striatal NPYcontaining neurons receive GABAergic afferents and may also contain GABA: a electron microscopic study in the rat. Eur. J. Neurosci. 2, Yamashita, A., Fuchs, E., Taira, M., and $672-681$.

Waldvogel, H. J., and Faull, R. L. M. (1993). Compartmentalization of parvalbumin immunoreactivity in the human striatum. Brain Res. $610,311-316$.

Wong, P., and Kaas, J. H. (2009). Architectonic subdivisions of neocortex in the tree shrew (Tupaia belangeri). Anat. Rec. (Hoboken) 292, 994-1027.

Wu, Y., and Parent, A. (2000). Striatal interneurons expressing calretinin, parvalbumin or NADPHdiaphorase: a comparative study in the rat, monkey and human. Brain Res. 863, 182-191.
Hayashi, M. (2010). Amyloid beta $(\mathrm{A} \beta)$ protein-and amyloid precursor protein (APP)-immunoreactive structures in the brains of aged tree shrews. Curr. Aging Sci. 3, 230-238.

Yelnik, J., Francois, C., Percheron, G., and Tande, D. (1991). Morphological taxonomy of the neurons of the primate striatum. J. Comp. Neurol. 313, 273-294.

Conflict of Interest Statement: The authors declare that the research was conducted in the absence of any commercial or financial relationships that could be construed as a potential conflict of interest.
Received: 25 September 2010; accepted: 01 August 2011; published online: 17 August 2011.

Citation: Rice MW, Roberts RC, Melendez-Ferro $M$ and Perez-Costas E (2011) Neurochemical characterization of the tree shrew dorsal striatum. Front. Neuroanat. 5:53. doi: 10.3389/fnana.2011.00053

Copyright (C) 2011 Rice, Roberts, Melendez-Ferro and Perez-Costas. This is an open-access article subject to a nonexclusive license between the authors and Frontiers Media SA, which permits use, distribution and reproduction in other forums, provided the original authors and source are credited and other Frontiers conditions are complied with. 